\title{
Die Weichbodengemeinschaft vor der Elbemündung unter dem Einfluß der Klärschlammverklappung. II. Die Gemeinschaftsstruktur im saisonalen Wechsel
}

\author{
Ute Mühlenhardt-Siegel \\ Zoologisches Institut und Zoologisches Museum der Universität Hamburg; \\ Martin-Luther-King-Platz 3, D-2000 Hamburg 13, Bundesrepublik Deutschland
}

\begin{abstract}
The soft bottom fauna near the River Elbe Estuary under the influence of sewage sludge. II. Seasonal changes in the community structure. Macrobenthic fauna and sediment parameters were studied at 40 stations representing a $494 \mathrm{~km}^{2}$ survey area in the eastern part of the German Bight and encompassing the sewage sludge dumping site. Data were taken in July 1980, one month after dumping ended, and in April 1981. $\mathrm{pH}$-value, redox potential of the interstitial water, amount of sand in the sediment as well as total number of species and diversity were lower in the dumping area than outside, whereas alkalinity and water content of the sediment were higher. Due to mass development of a few species around the dumping site, biomass and number of individuals were high in July. Abundance and biomass values were lower in April. 2 communities are described and related to abiotic parameters such as sediment structure. Due to disturbance effects of sewage sludge, 2 stages of the second successional phase (Pearson \& Rosenberg, 1978) were ascertained, described by one of these communities each time. The first faunal community, characterized by an Abra alba assemblage, describes an early stage of the second successional phase. In summer (July) the Abra alba community showed a wider spatial range than in spring. The other, dominated by Nucula nitida, indicated a later stage of the second succession phase that was passing to the third phase.
\end{abstract}

\section{EINLEITUNG}

Während in Teil 1 dieser, Untersuchung beispielhaft an 5 Dauerstationen (Abb. 1) und einer Vergleichsstation saisonale Fluktuationen von Individuenzahl, Artenzahl und Biomasse unter dem Einfluß der Klärschlammverklappung untersucht wurden, soll hier die Benthosgemeinschaft im Einflußbereich der Klärschlammeinbringung mit der umliegender Gebiete verglichen werden.

\section{UNTERSUCHUNGSGEBIET UND METHODE}

Im Juli 1980 und April 1981 wurden in einem Stationsnetz von 40 Stationen je 2 Parallelproben genommen (Abb. 1). Die Stationen lagen im Schlickgebiet der Helgoländer Bucht (Figge, 1981) in einem Areal von $494 \mathrm{~km}^{2}$. Die Wassertiefe nahm von Osten nach Westen gleichmäßig von $13 \mathrm{~m}$ bis $32 \mathrm{~m} z u$.

Sedimentanalysen und Bearbeitung des Tiermaterials erfolgten wie in Teil 1 beschrieben (Mühlenhardt-Siegel, 1988). Vom Deutschen Hydrographischen Institut standen Angaben über Alkalinität, pH-Wert und Redoxpotential des Oberflächensedi- 


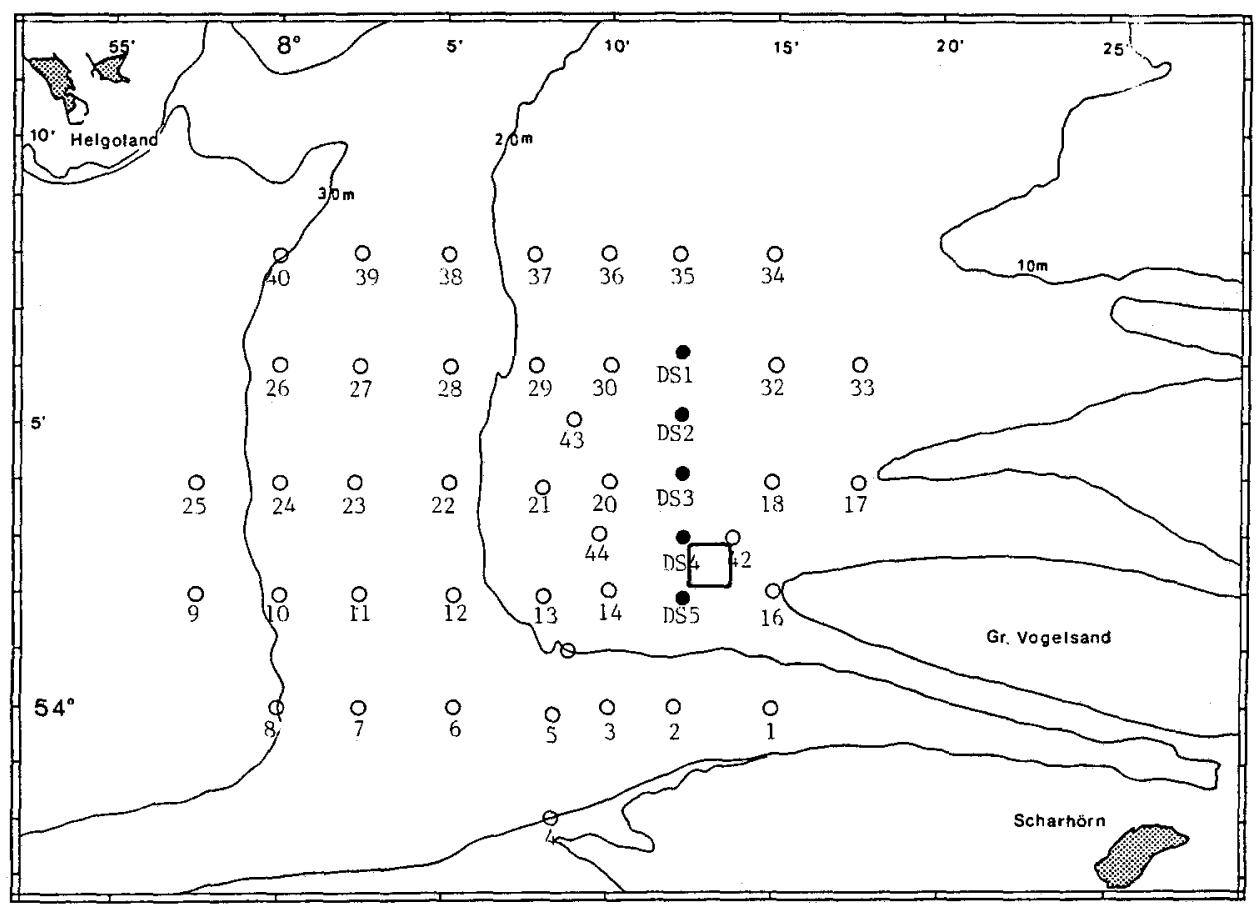

Abb. 1. Die Stationen im Untersuchungsgebiet. Die Dauerstationen sind mit DS1-DS5 gekennzeichnet. Das Quadrat markiert das Einbringungsgebiet

mentes zur Verfügung. Die Biomasse wurde als aschefreies Trockengewicht (AFTG) angegeben.

Um Stationen nach Ähnlichkeiten bezüglich der faunistischen Zusammensetzung zusammenzufassen, wurde eine Clusteranalyse berechnet (Mirza \& Gray, 1981). Als Maß für die Ähnlichkeit jeweils zweier Stationen wurde die Euklidische Distanz D gewählt (Stephenson, 1972).

Für zwei Stationen $\mathrm{x}_{1}$ und $\mathrm{x}_{2}$ ist die Distanz $\mathrm{D}$ für $\mathrm{m}$ Merkmale (hier: $\mathrm{m}=$ Arten) definiert als:

$$
D_{1 / 2}=\sqrt{\Sigma\left(x_{1 j}-x_{2 j}\right)^{2}} \text { mit } j=1 \text { bis m }
$$

Mit Hilfe des Distanzmaßes werden Stationen in Gruppen zusammengefaßt, die sich bezüglich der gewählten Eigenschaften, in diesem Falle Arten, ähneln.

Die Berechnung erfolgte mit dem CLUSTAN 2 Programmpaket am Rechenzentrum der Universität Hamburg.

Die Diversität (Shannon-Wiener-Index) wurde auf der Basis ln berechnet, die Evenness nach Pielou (1974).

Um die Korrelationen zwischen den Abundanzen der auftretenden Arten zu erkennen, wurde die Rangkorrelation nach Spearman (Sachs, 1984) berechnet. Hierfür wurde das SPSS 9 Programmpaket verwendet. Die Rangkorrelation nach Spearman ist als verteilungsfreies Verfahren bei nicht normalverteilten Werten zu verwenden. Getestet 
wurden alle Arten gegeneinander, die im Juli 1980 und April 1981 jeweils gefunden wurden.

Zwischen den abiotischen und biotischen Parametern jeder Probe wurden Regressionen berechnet und mit Hilfe des F-Tests (Sachs, 1984) auf ihre Signifikanz geprüft. Korrelationen wurden als signifikant gewertet, wenn sie sich auf dem $5 \%$-Niveau absichern ließen.

\section{ERGEBNISSE}

\section{Abiotische Faktoren}

Neben Korngröße und Wassergehalt wurden im Untersuchungsgebiet die sedimentchemischen Parameter pH-Wert, Alkalinität und Redoxpotential gemessen:

p H - W e r t. Der pH-Wert der aufgeschlämmten Oberflächensedimente lag im Juli 1980 zwischen 7,37 und 8,78. Der Bereich des niedrigsten $\mathrm{pH}$ lag in einem kleinen Zentrum westlich des Verklappungsgebietes und nahm nach allen Seiten hin zu. Im April 1981 waren 3 Zonen mit niedrigen (unter 8) pH-Werten zu bemerken, wovon der geringste 7,37 war. Von diesen Zonen stieg der Wert nach allen Seiten hin an. Auf einem höheren pH-Niveau war nur noch ein isoliertes Areal mit $\mathrm{pH}=8,25$ festzustellen, das von Norden nach Süden durch Bereiche mit niedrigem pH eingeengt wurde (Abb. 2).

Alkalinität. Die Alkalinität im Porenwasser der Oberflächensedimente wurde erstmalig im April 1981 im Untersuchungsgebiet gemessen. Die Bereiche höchster Alkalinität lagen im April 1981 im NW des ehemaligen Klärschlammverklappungsgebietes und erreichten maximale Werte bis 23 meqv $1^{-1}$ (Milliaequivalent pro Liter). Von einem kleinen Zentrum aus nahm dieser Wert nach allen Seiten hin ab. Vor allem nach Norden und Süden erstreckten sich "Zungen“ mit erhöhter Alkalinität (Abb. 3).

Redoxp ot ential. Das Redoxpotential der Oberflächensedimente in $3 \mathrm{~cm}$ Tiefe des Sedimentes hatte die niedrigsten Werte im Juli 1980 (unter -200 eh in mV) westlich des Verklappungsgebietes und an 2 Stationen am Nordrand des Untersuchungsgebietes. Zu den Rändern des Gebietes hin nahm das Redoxpotential zu. Der höchste Wert lag an der SE-Ecke des großen Stationsnetzes an einer Station nahe der Fahrrinne mit $+100 \mathrm{mV}$ (Abb. 4).

Korngröße und Wa s s e rge halt. Eine Korngrößenanalyse ließ für Juli 1980 ein kleines Zentrum mit minimalem Anteil der größeren Korngrößen (über $31 \mu \mathrm{m}$ ) westlich der Dauerstationen erkennen, von dem aus der gröbere Anteil nach allen Seiten hin zunahm, was durch die Nähe der benachbarten Sände gut zu erklären ist (Abb. 5). Der Wassergehalt der Sedimente war stark mit den kleinsten Korngrößen korreliert (Mühlenhardt-Siegel, 1988). Im Juli 1980 entsprach die Verbreitung von hohem Wassergehalt der der feinen Korngrößen. Im Vergleich dazu hatte sich im April 1981 der Bereich mit hohem Porenwasseranteil ausgedehnt, so daß auch an den nördlichsten Stationen wasserreiche Sedimente vorlagen. Es zeigte sich, daß vor allem in der Nähe des ehemaligen Klärschlammverklappungsgebietes die Stationen an Porenwasser verloren hatten, während die Stationen im Norden und Westen einen erhöhten Wasseranteil hatten.

Die Änderungen aller abiotischen Faktoren erfolgten stets nach Osten über eine kürzere Distanz als nach Westen. 

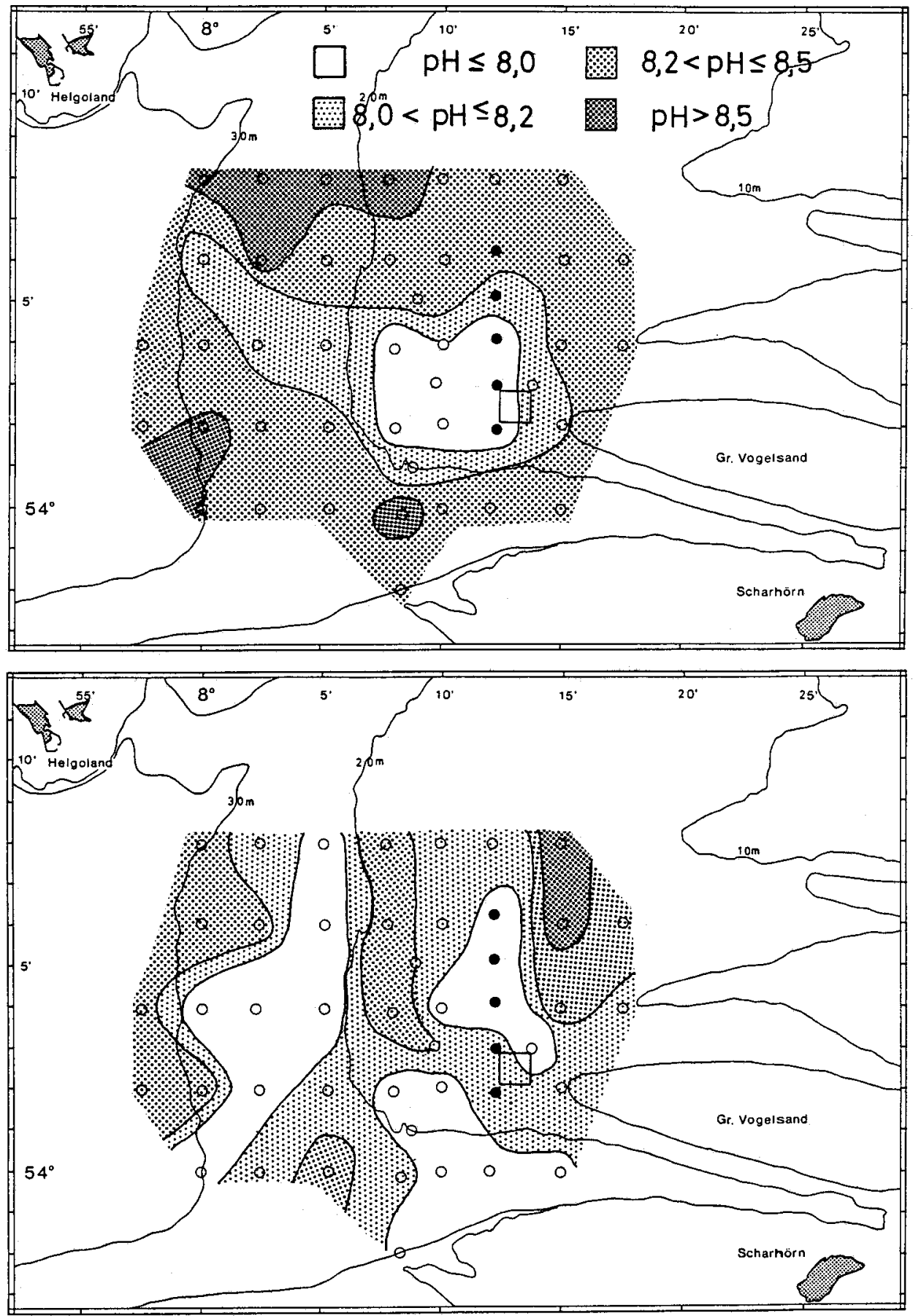

Abb. 2. Die Verteilung des pH-Wertes im Juli 1980 (oben) und April 1981 (unten) 


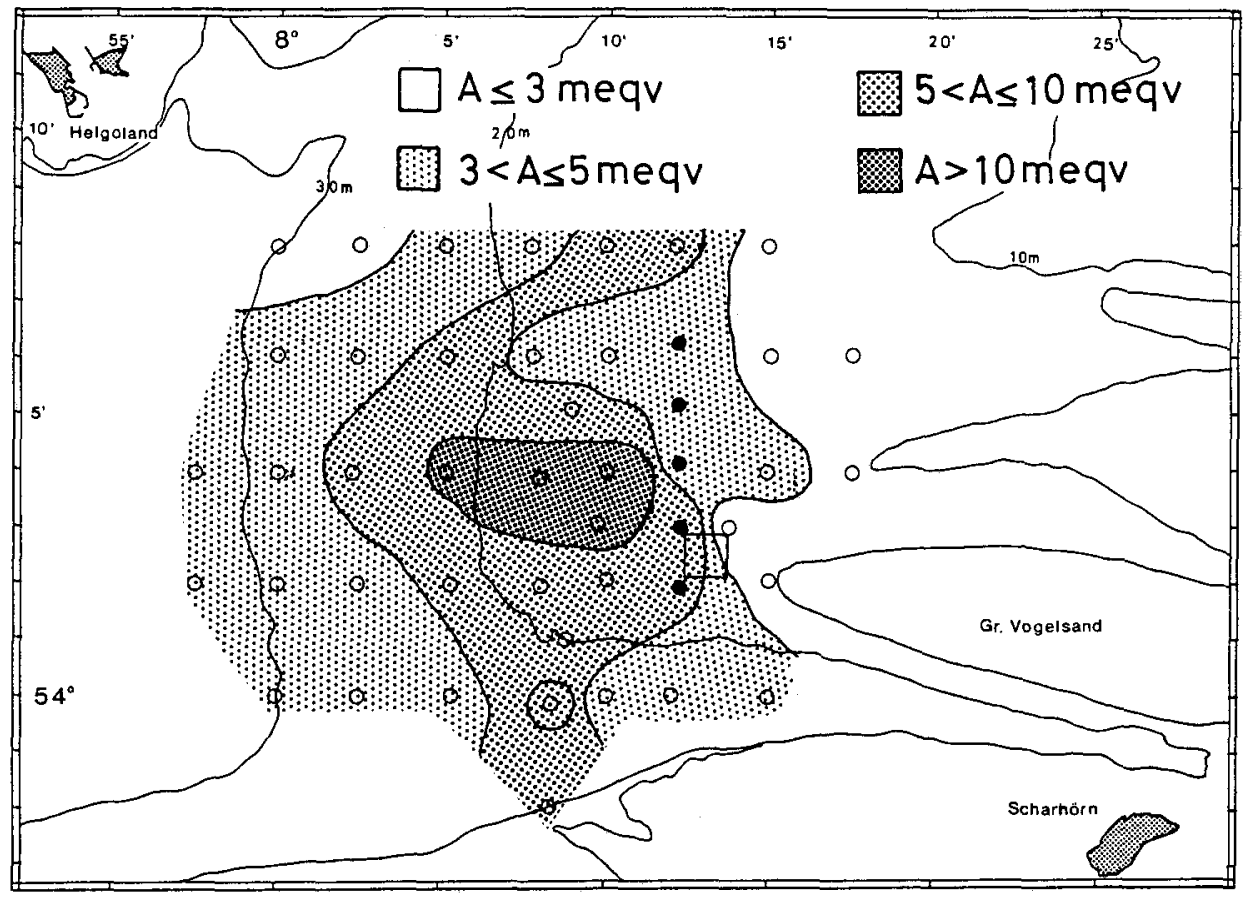

Abb. 3. Die Verteilung der Alkalinität (A) in meqv (Milliaequivalent pro Liter) im April 1981

\section{Arten- und Individuenzahl}

Die Artenzahl an den Stationen des Untersuchungsgebietes wurde im Juli 1980 stark beeinflußt durch Massenentwicklungen einzelner Arten gegenüber den Vormonaten und -jahren (Mühlenhardt-Siegel, 1981, 1988). Hierbei fiel vor allem Abra alba auf. Die Massenentwicklung dieser Art (maximal $17120 \mathrm{Ind}^{-2} \mathrm{~m}^{-2}$ ) lag in der Nähe des Klärschlammverklappungsgebietes (Abb. 6). Die Artenzahl war im Bereich des Einbringungsgebietes und westlich davon gering gegenüber dem Rand des Untersuchungsgebietes (Abb. 7). Dieser Bereich deckte sich weitgehend mit der Zone der Abra albaMassenentwicklung. Die höchste Artenzahl war mit über 40 Arten am Nord- und Südwestrand des Untersuchungsgebietes anzutreffen. Insgesamt wurden im Juli 198092 Arten gefunden (Mühlenhardt-Siegel, 1985). Hiervon wurden 34 Arten an mehr als einem Drittel aller Stationen angetroffen (Tab. 1).

Im April 1981 war die Abra alba-Population weitgehend zusammengebrochen (Abb. 6). Allein an zwei Stationen war die Individuenzahl dieser Art noch relativ hoch mit maximal 4100 Ind. $\mathrm{m}^{-2}$. Auch die Artenzahl war im Frühling gegenüber dem Vorsommer zurückgegangen. Die Änderung in der Artenzahl hatte vor allem in den Randgebieten des Untersuchungsgebietes stattgefunden (Abb. 7). Die niedrigste Artenzahl (10 Arten) war innerhalb eines kleinen Bereichs westlich des ehemaligen Verklappungsgebietes anzutreffen. Die höchste Artenzahl (32 Arten) wurde an einer Station am Südwestrand des Untersuchungsgebietes gefunden. Im April wurden 72 Arten gefunden. Auch hatte im Vergleich zum Juli 1980 die Präsenz der Arten abgenommen (Tab. 1). 

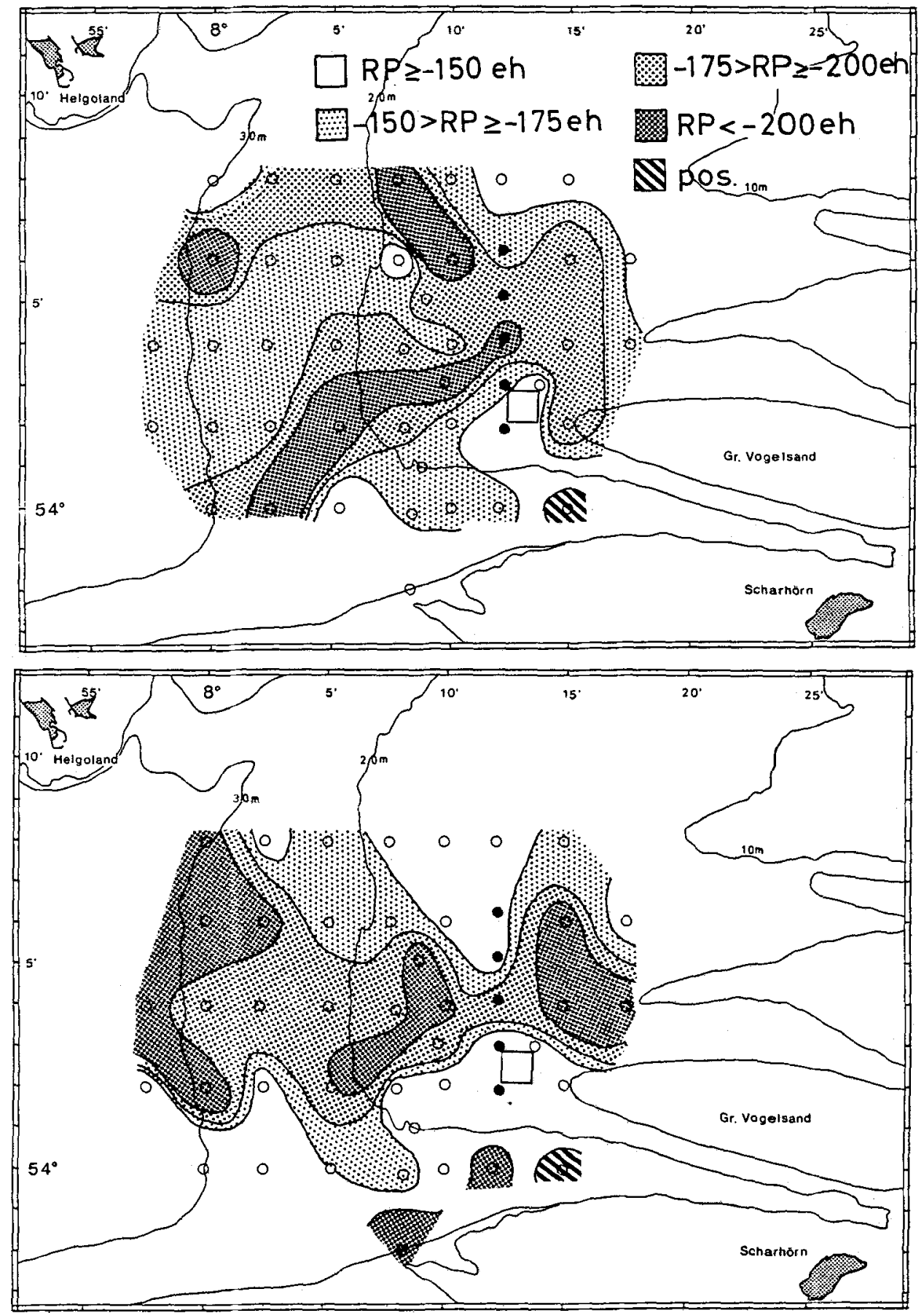

Abb. 4. Die Verteilung des Redoxpotentials (RP) im Juli 1980 (oben) und April 1981 (unten) 


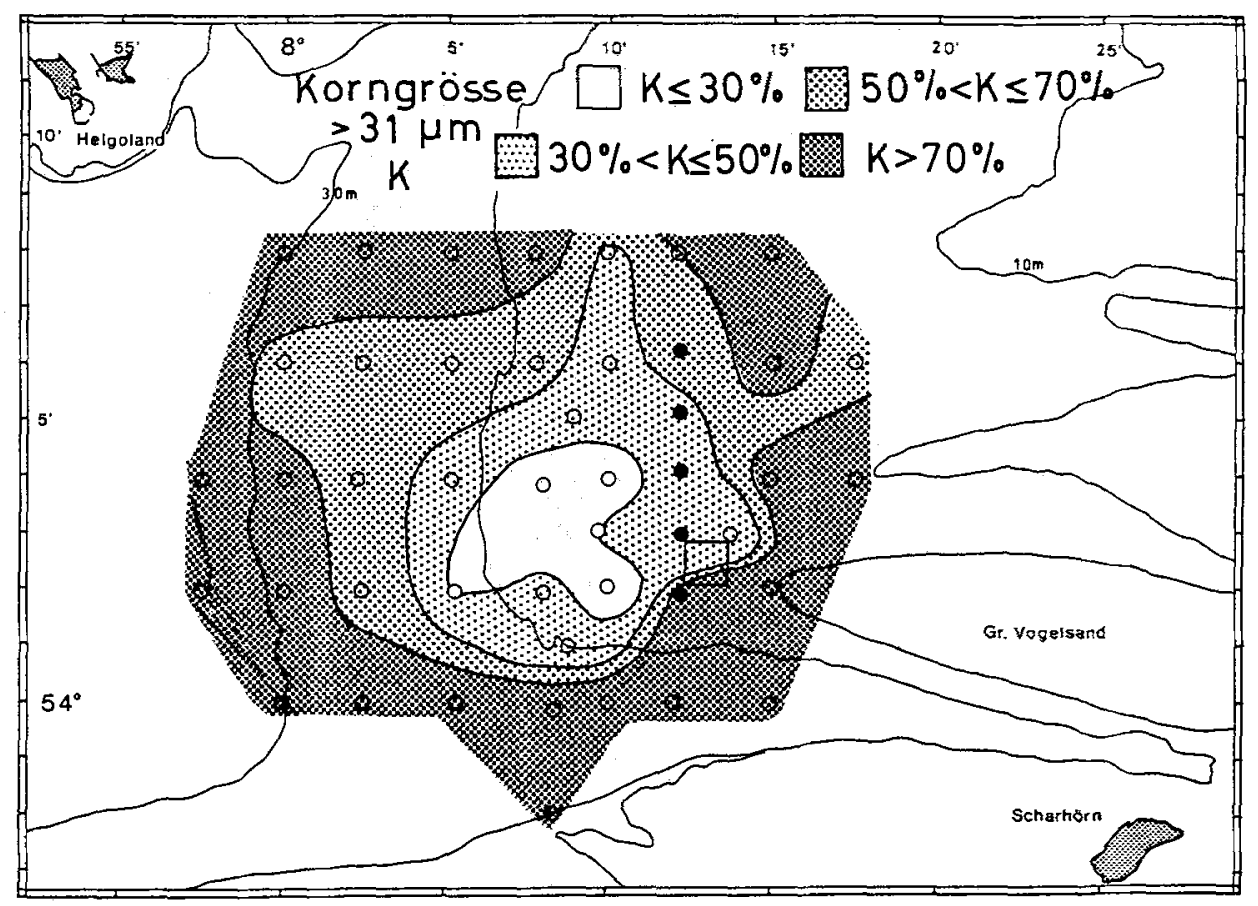

Abb. 5. Die Korngrößenverteilung der Fraktion über $31 \mu \mathrm{m}(\mathrm{K})$ im Juli 1980

Folgende Arten fehlten im April: Harmothoe longisetis, Gattyana cirrosa, Anaitides subulifera, Nereis longissima, Polydora pulchra, P. ligni, Sthenelais boa, Chone duneri, Arenicola marina, Cardium edule, Petricola pholadiformis, Cylichna cylindracea, Mya truncata, Melita obtusata, Nototropis falcatus, Microprotopus maculatus, Stenothoe marinus, Orchomenella nana, Corophium volutator, Echiurus echiurus, Anthozoa sp., Pantopoda sp. und juvenile Plattfische.

Nur im April 1981 wurden folgende Arten gefangen: Harmothoe imbricata, Venus gallina, Cyprina islandica, Buccinum undatum, Bathyporeia pilosa, Megaluropis aegilis, Nototropis vedlomensis, Calianassa stebbingi und Synchelidium haplocheles.

Die Individuendichte nahm in der Regel mit zunehmender Entfernung von der Küste hin ab. Die höchste Individuendichte lag im Juli 1980 nördlich des Großen Vogelsandes mit 46750 Ind. $\mathrm{m}^{-2}$ (Abb. 8). Die hohen Individuenziahlen wurden vor allem verursacht durch starkes Auftreten der Arten Abra alba, Ensis directus, Scalibregma inflatum und Scoloplos armiger.

Im April 1980 fand die Probennahme zur Zeit des Auftretens juveniler Diastylis rathkei statt. Wurden diese nicht berücksichtigt, zeigte sich ein ansonsten individuenarmer Streifen in Küstennähe sowie einer in Verlängerung der Elbemündung. Letzterer wurde flankiert von individuenreicheren Gebieten im Norden und Süden (Abb. 8). Die dominanten Arten im Untersuchungsgebiet und -zeitraum waren Albra alba, Nucula nitida und Diastylis rathkei. 

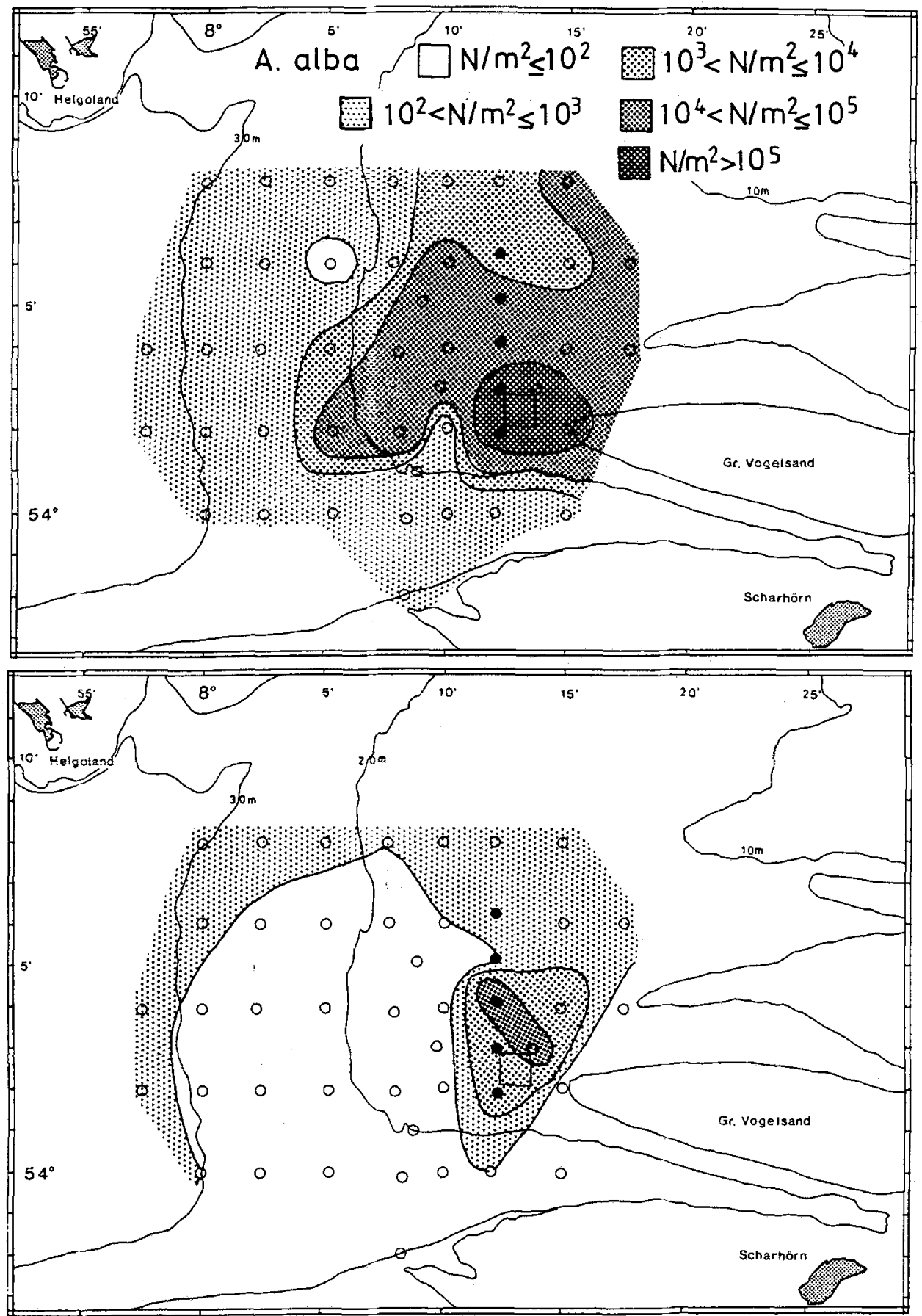

Abb. 6. Die Individuenzahl (N) pro Quadratmeter von Abra alba im Juli 1980 (oben) und April 1981 (unten) 

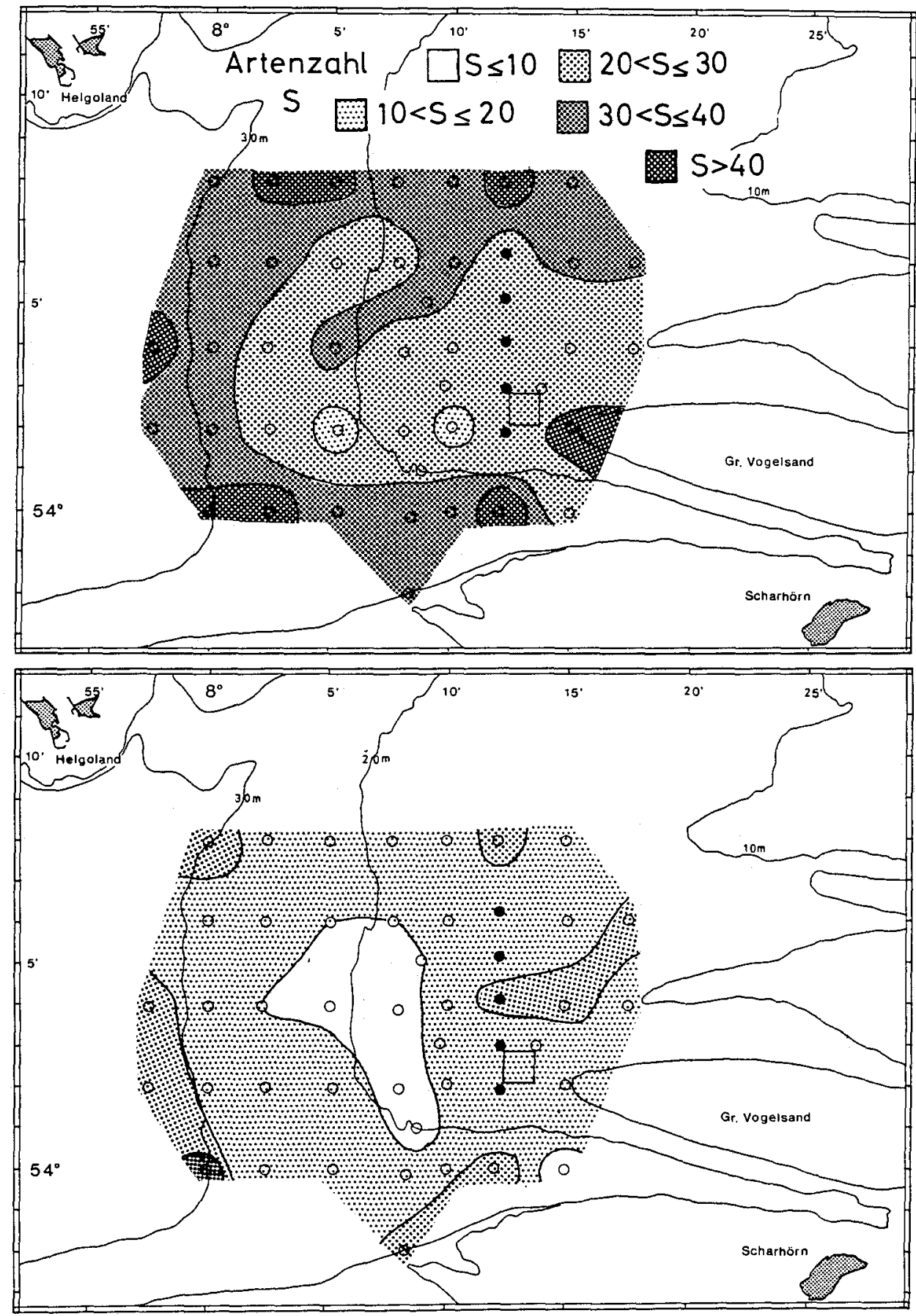

Abb. 7. Die Verteilung der Artenzahl (S) im Juli 1980 (oben) und April 1981 (unten) 
Tabelle 1. Präsenz der häufigen Arten. Angaben in \% der Proben (n=80)

\begin{tabular}{|c|c|c|c|c|c|}
\hline \multicolumn{5}{|c|}{ Juli 1980} & \\
\hline Anaitides groenlandica & 100 & Anaitides juv. & 69 & Edwardsia sp. & 49 \\
\hline Nephtys hombergi & 99 & Notomastus latericeus & 51 & Cerianthus lloydii & 45 \\
\hline Diastylis rathkei & 99 & Gattyana cirrosa & 50 & Lineus sp. & 43 \\
\hline Scalibregma inflatum & 99 & Ophiura albida & 50 & Ophelia acumenata & 43 \\
\hline Nucula nitida & 95 & & & Nephtys caeca & 43 \\
\hline Eteone sp. & 95 & & & Tellina sp. & 41 \\
\hline Spiophanes bombyx & 91 & & & Crangon crangon & 39 \\
\hline Pholoe minuta & 89 & & & Ampharete sp. & 39 \\
\hline Scoloplos armiger & 89 & & & Macoma baltica & 39 \\
\hline Pectinaria koreni & 87 & & & Harmothoe lunulata & 38 \\
\hline Abra alba & 86 & & & Halcampa sp. & 38 \\
\hline \multirow[t]{9}{*}{ Ophiura texturata } & 80 & & & Mysella bidentata & 37 \\
\hline & & & & Ampelisca brevicornis & 37 \\
\hline & & & & Portunus holsatus & 37 \\
\hline & & & & Echinocardium cordatum & 36 \\
\hline & & & & Lanice conchilega & 35 \\
\hline & & & & Ensis directus & 35 \\
\hline & & & & Diastylis bradyi & 34 \\
\hline & & & & Goniada maculata & 28 \\
\hline & & & & Eumida punctifera & 26 \\
\hline \multicolumn{6}{|c|}{ April 1981} \\
\hline$\geqslant 75 \%$ & & \multicolumn{2}{|l|}{$50-<75 \%$} & \multicolumn{2}{|l|}{$25-<50 \%$} \\
\hline Nephtys hombergi & 96 & Scoloplos armiger & 69 & Abra alba & 46 \\
\hline Nucula nitida & 90 & Ophiura texturata & 57 & Nephtys caeca & 45 \\
\hline Diastylis rathkei & 90 & Pholoe minuta & 56 & Anaitides groenlandica & 37 \\
\hline & & & & Macoma baltica & 29 \\
\hline & & & & Mysella bidentata & 29 \\
\hline
\end{tabular}

\section{Diversität}

Wie erwartet war bei der Massenentwicklung weniger Arten im Juli 1980 die Diversität am geringsten nordöstlich und westlich des Verklappungsgebietes, wo Ensis directus (östlich) und Abra alba (westlich) häufig waren (Abb. 9a). Mit Ausnahme der westlichsten Station war bei geringer Diversität auch die Evenness am niedrigsten (Abb. 9b), die Individuenzahl war also sehr ungleichmäßig auf die einzelnen Arten verteilt. Im Vergleich zum Juli 1980 hatten sich im April 1981 der Bereich der geringsten Diversität und Evenness nach Westen und Nordwesten verlagert.

\section{Biomasse}

Die Biomasse (AFTG) wurde im Juli 1980 stark durch die Massenentwicklung von Abra alba beeinflußt. Besonders hohe Werte fanden sich an den Stationen in der Nähe des Klärschlammverklappungsgebietes. Ein weiteres Gebiet mit hoher Biomasse war am 

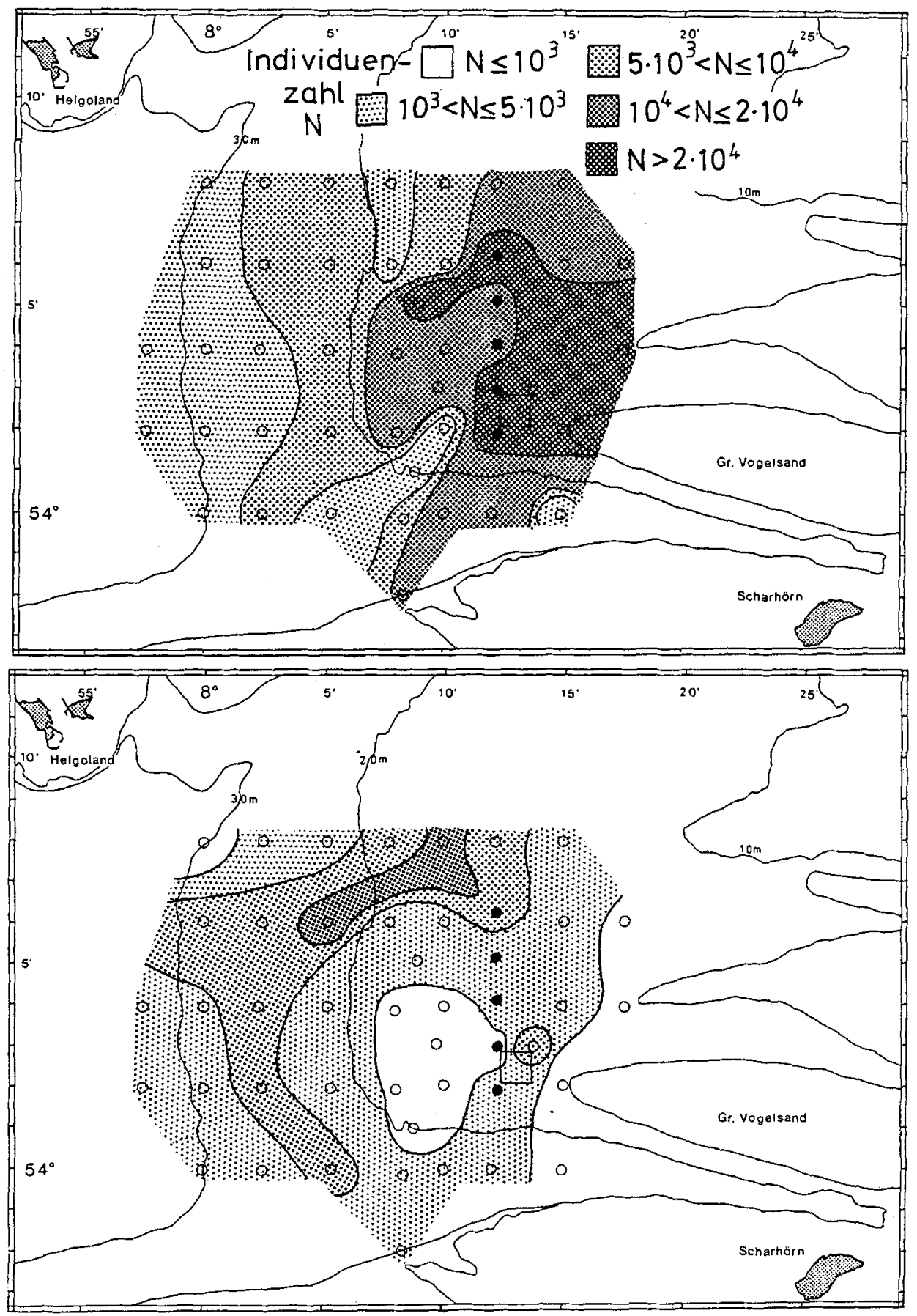

Abb. 8. Die Verteilung der Individuenzahl (N) pro Quadratmeter im Juli 1980 (oben) und April 1981 (unten) 

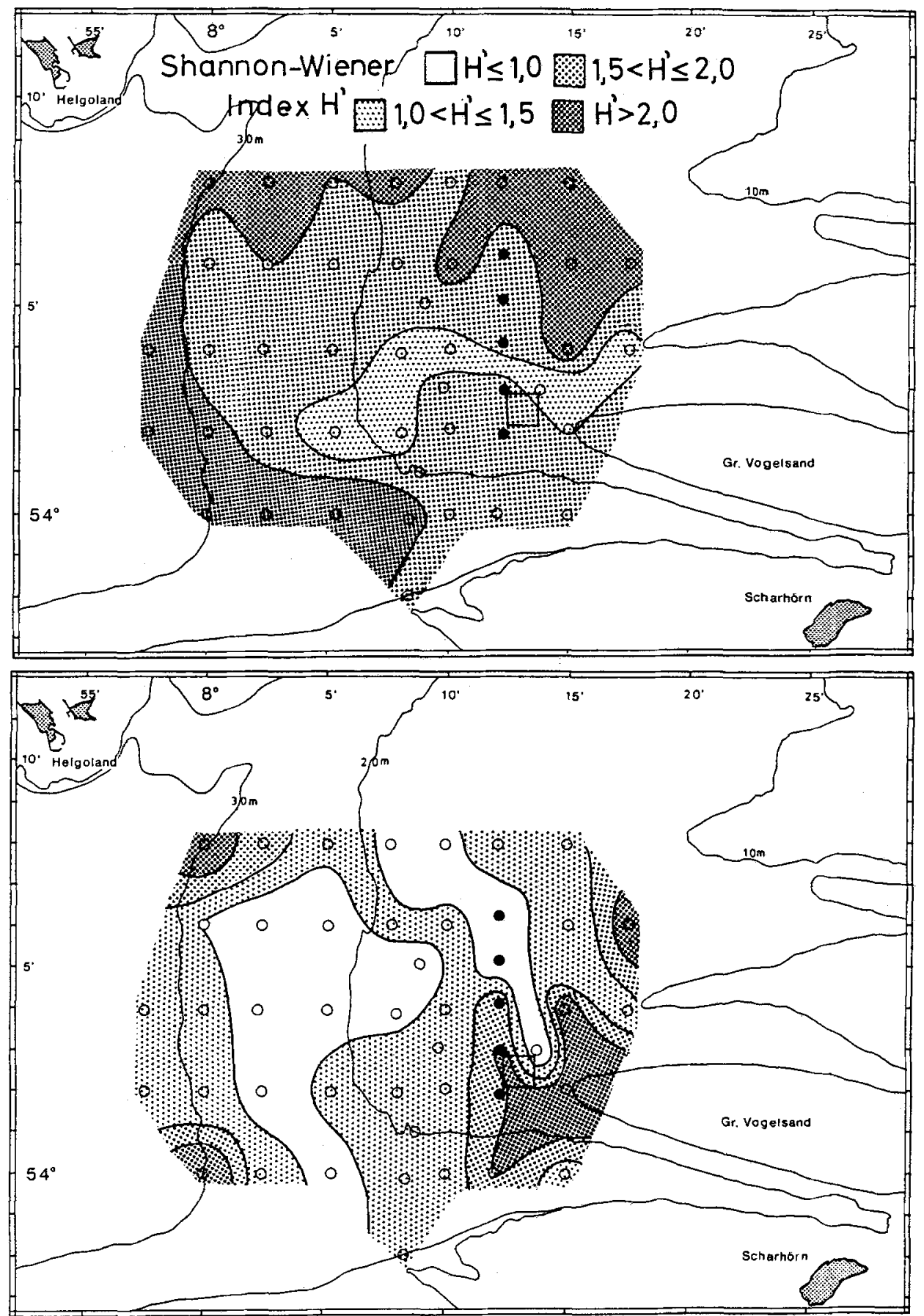

Abb. 9a. Die Verteilung der Diversität im Juli 1980 (oben) und April 1981 (unten): Shannon-WienerIndex $\left(\mathrm{H}^{\prime}\right)$ 

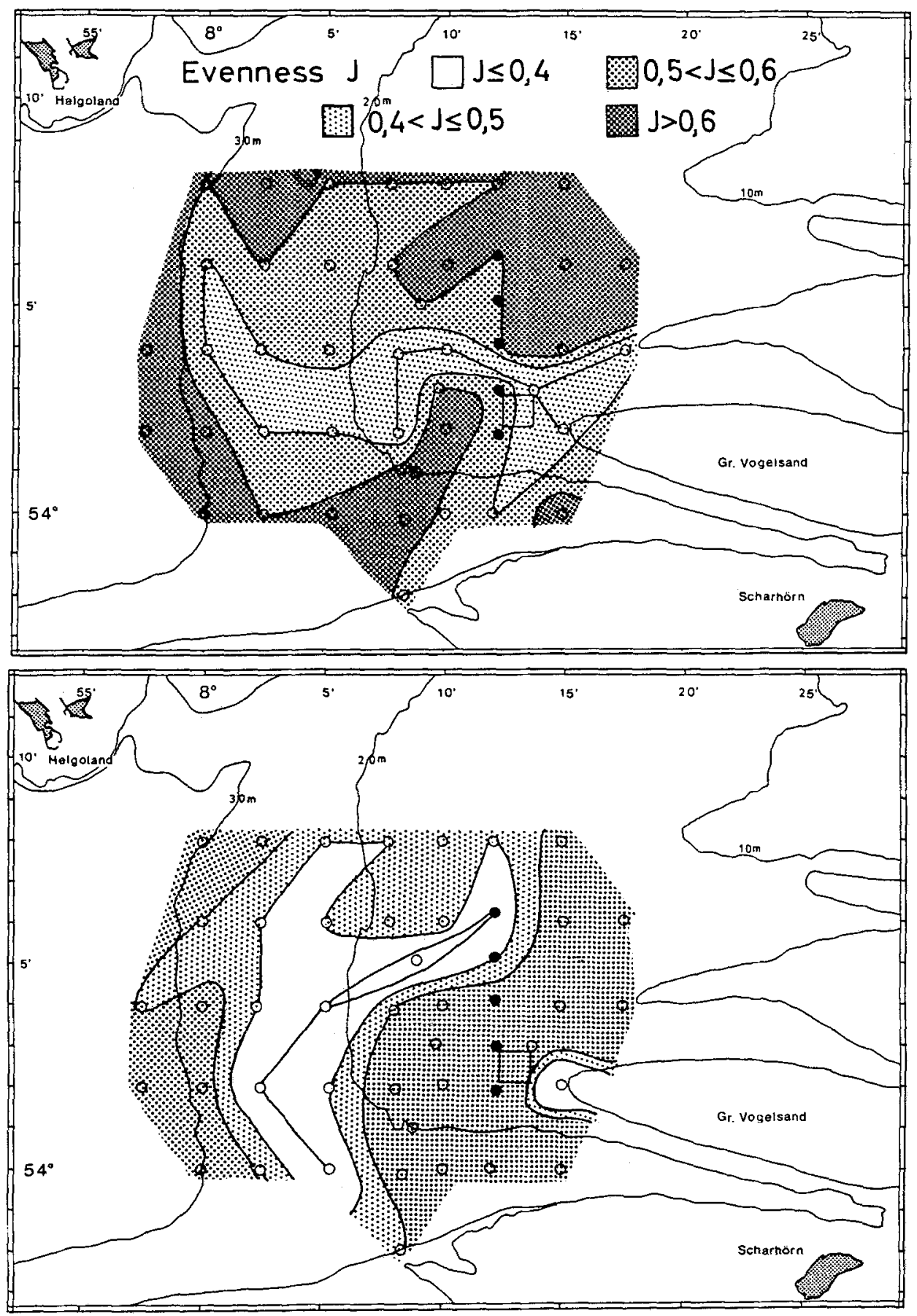

Abb. 9b. Die Verteilung der Diversität im Juli 1980 (oben) und April 1981 (unten): Evenness (J) 

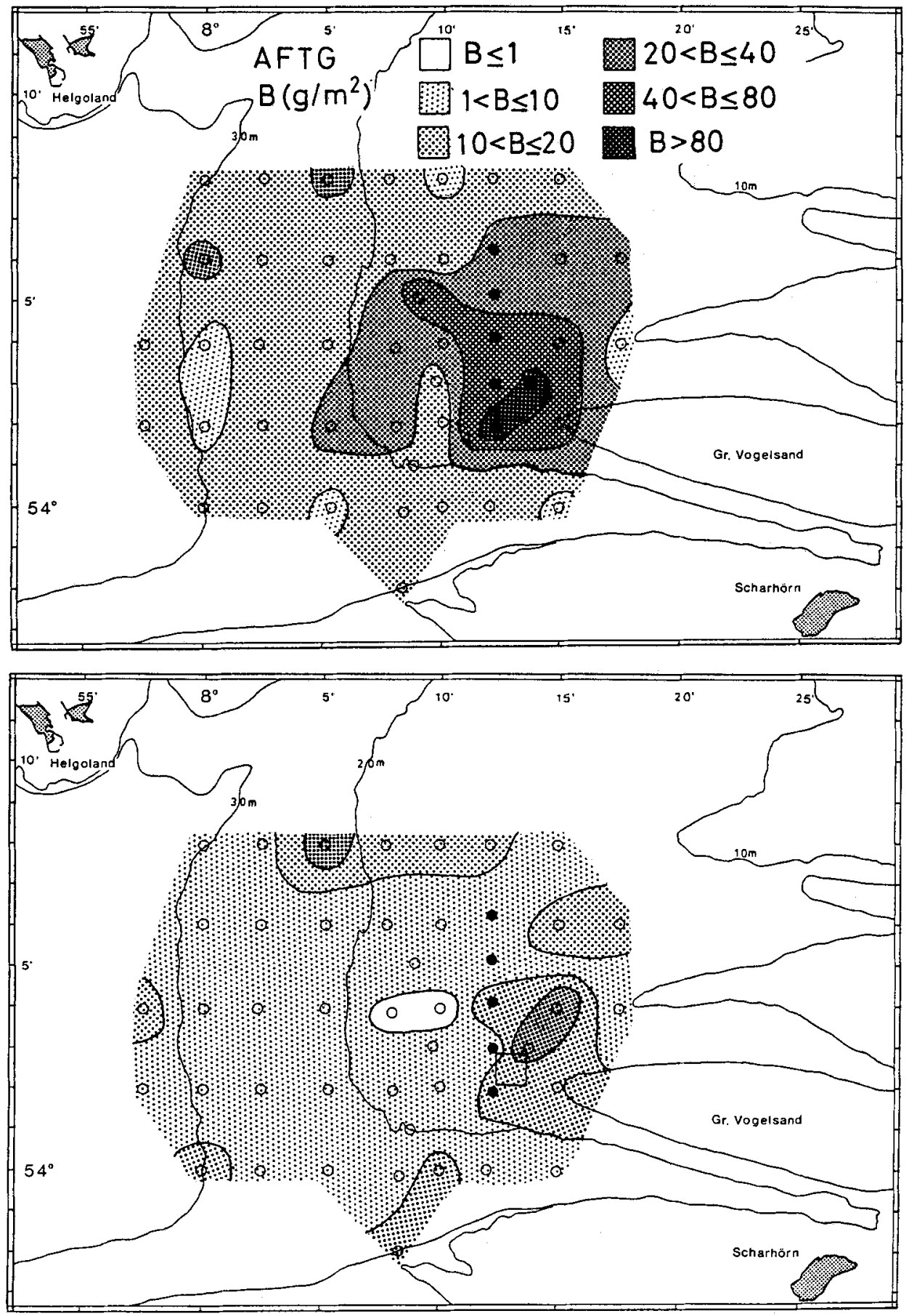

Abb. 10. Die Verteilung der Biomasse B (AFTG) im Juli 1980 (oben) und April 1981 (unten) 
Nordwestrand des Probennahmegebietes zu finden (Abb. 10). Die Arten Abra alba und Nucula nitida machten in weiten Bereichen über $50 \%$ der Biomasse aus. Die höchste Biomasse wurde an Stationen gemessen, an denen Abra alba allein über $75 \%$ der Gesamtbiomasse repräsentierte. Das zweite Gebiet mit hoher Biomasse lag im Bereich der Stationen, wo Nucula nitida 25 bis $50 \%$ und Diastylis rathkei über $20 \%$ Anteil an der Gesamtbiomasse hatten.

Im April 1981 war die Biomasseverteilung gegenüber dem Juli 1980 verändert. Die geringsten Werte wurden nordwestlich des ehemaligen Klärschlammverklappungsgebietes gemessen (Abb. 10), während die höchsten Werte am Ostrand des Untersuchungsgebietes zu finden waren. Auch im April erreichten die häufigsten Arten Abra alba, Núcula nitida und Diastylis rathkei in weiten Teilen des Untersuchungsgebietes zusammen über $50 \%$ der Gesamtbiomasse. Die Gewichtsdominanz hatte sich aber im Vergleich zum Juli des Vorjahres verändert: Abra alba stellte im April 1981 nur noch an 4 Stationen 25 bis $50 \%$ des Gesamtgewichts, während Nucula nitida im Vergleich zum Juli 1980 höhere Gewichtsanteile erlangt hatte. Diastylis rathkei hatte im April nur wenig Anteil am Gesamtgewicht.

Die Abnahme der Biomasse vom Juli 1980 bis April 1981 war am höchsten in einem Gebiet nordwestlich des ehemaligen Klärschlammverklappungsgebietes (Abb. 11). Zu den Rändern des Untersuchungsgebietes hin war die Abnahme der Biomasse geringer, am Nordrand war sogar eine Zunahme zu registrieren.

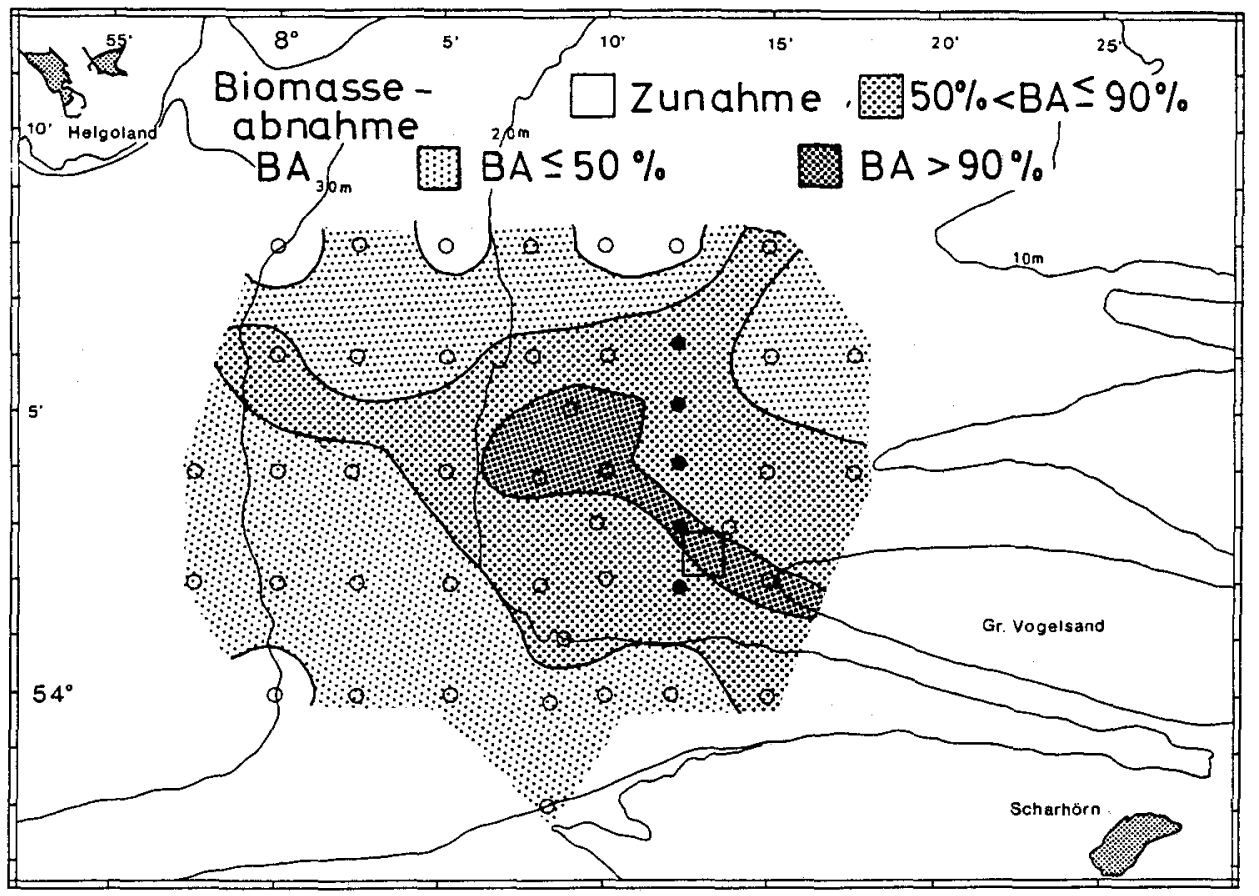

Abb. 11. Die Verteilung der Biomasse-Veränderung (BA) von Juli 1980 zum April 1981 in Prozent 


\section{Korrelation von Artenzahl und Biomasse einzelner Taxa zu den abiotischen Faktoren}

Die signifikanten Korrelationen $(P \leq 0,05)$ zwischen Artenzahl bzw. Biomasse einzelner Taxa und den sedimentchemischen Parametern pH-Wert, Alkalinität und Redoxpotential sind in Tabelle 2 zusammengefaßt.

Tabelle 2. Signifikante Korrelationen $(\mathrm{P} \leq 0,05)$ zwischen Artenzahl bzw. Biomasse und abiotischen Faktoren, angegeben durch den Korrelationskoeffizienten $(n=40) ; \alpha=$ ohne $N$. hombergi und $P$. Koreni, $\beta=$ ohne $D$. rathkei, $\sigma=$ ohne $A$. alba und $N$. nitida

\begin{tabular}{|c|c|c|c|c|c|}
\hline & \multicolumn{2}{|c|}{$\mathrm{pH}$} & \multicolumn{2}{|c|}{ Redox } & \multirow{2}{*}{$\begin{array}{c}\text { Alkalinitä } \\
\text { April }\end{array}$} \\
\hline & Juli & April & Juli & April & \\
\hline \multicolumn{6}{|l|}{ Artenzahl } \\
\hline Polychaeta & $+0,44$ & & & & $-0,60$ \\
\hline Amphipoda & $+0,30$ & & & & \\
\hline Mollusca & $-0,33$ & & & $-0,43$ & $-0,75$ \\
\hline Echinodermata & $+0,49$ & & & & $-0,66$ \\
\hline Gesamtartenzahl & $+0,52$ & & & & $-0,61$ \\
\hline \multicolumn{6}{|l|}{ Biomasse } \\
\hline$N$. hombergi & $+0,35$ & & & $-0,41$ & $-0,66$ \\
\hline P. koreni & $-0,42$ & & $-0,36$ & & \\
\hline Polychaeta $\alpha$ & & $-0,47$ & & & $-0,64$ \\
\hline D. rathkei & $+0,40$ & & $-0,58$ & & \\
\hline Crustacea $\beta$ & & & & & $+0,40$ \\
\hline A. alba & $-0,61$ & & & & \\
\hline N. nitida & $+0,47$ & & $-0,34$ & $-0,36$ & $-0,56$ \\
\hline Mollusca $\sigma$ & $-0,40$ & & & & $-0,35$ \\
\hline O. texturata & & & $+0,37$ & & $-0,61$ \\
\hline Gesamtbiomasse & $-0,53$ & & $-0,34$ & & $-0,73$ \\
\hline
\end{tabular}

Mit Ausnahme der Mollusca waren im Juli 1980 die Artenzahlen aller Taxa positiv mit dem pH-Wert korreliert. Auffallend war daß im Frühling keine Beziehung der Artenzahlen zum pH-Wert nachgewiesen werden konnte. Signifikante Beziehungen zum Redoxpotential hatte nur die Artenzahl der Mollusca im April 1981. Zur Alkalinität waren die Artenzahlen negativ korreliert.

Die Biomasse der Arten Nephtys hombergi, Diastylis rathkei und Nucula nitida war positiv mit dem $\mathrm{pH}$-Wert korreliert. Die anderen häufigen Arten und übrigen Taxa zeigten negative Beziehungen zum pH-Wert. Mit Ausnahme der Polychaeta (ohne Nephtys hombergi und Pectinaria koreni) waren nur für Juli 1980 signifikante Korrelationen nachzuweisen. Abgesehen von Ophiura texturata war die Biomasse aller Taxa negativ mit dem Redoxpotential korreliert. Wie die Artenzahl war auch die Biomasse in der Regel negativ mit der Alkalinität in Beziehung zu setzen. Hier bildeten nur die Crustacea ohne Diastylis rathkei eine Ausnahme. 


\section{Korrelationen der Artenabundanzen}

Um zu erkennen, wie die einzelnen Arten im gesamten Untersuchungsgebiet zueinander in Beziehung stehen, wurde für die Artenabundanzen beider Probennahmen eine Rangkorrelation nach Spearman (Sachs, 1984) berechnet. Die Korrelationen der einzelnen Arten zueinander sind an anderer Stelle detailliert aufgeführt (Mühlenhardt-Siegel, 1985). Auf Grund der Übereinstimmung ihrer Begleitfauna lassen sich für Juli 1980 und April 1981 die häufigsten Arten wie in Tabelle 3 dargestellt gruppieren.

Tabelle 3. Die Hauptarten und ihre Begleitfauna nach der Rangkorrelation von Spearman.

- $=$ Hauptarten, $+=$ positive Korrelation, $-=$ negative Korrelation $(P \leq 0,05)$

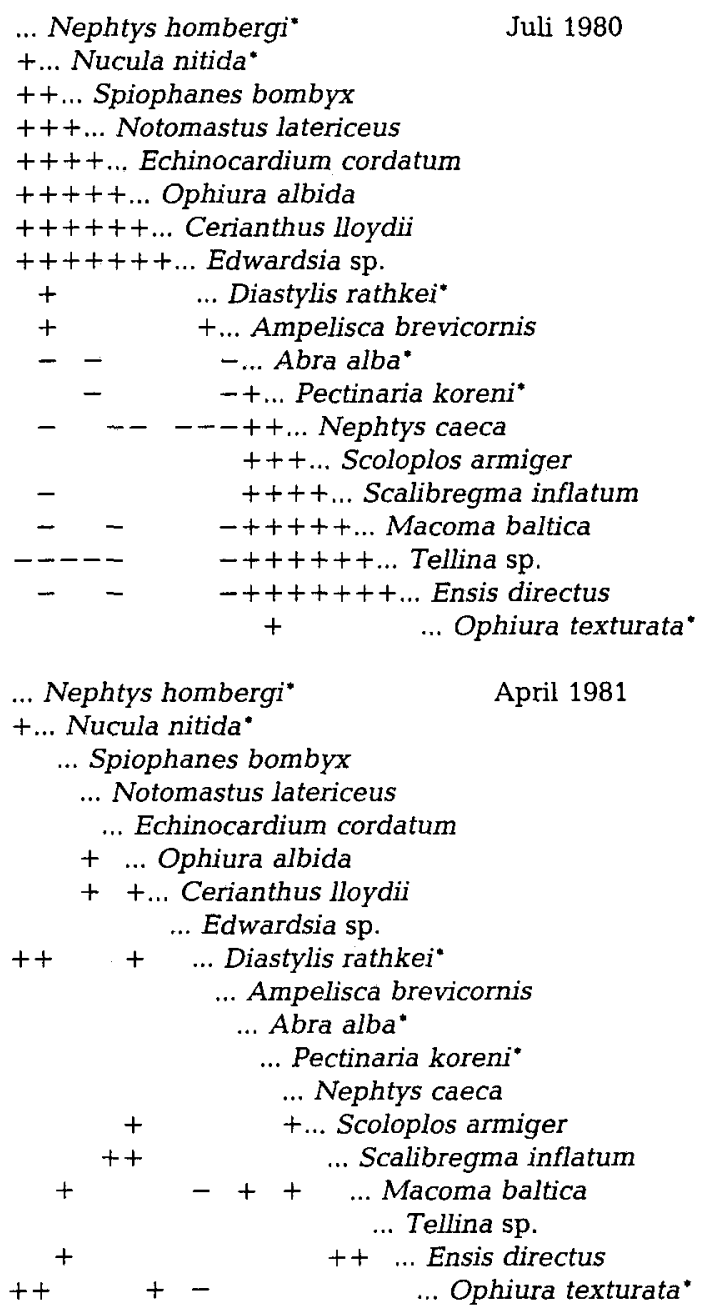




\section{Zusammenfassung der Stationen nach Artengemeinschaften}

Für Juli 1980 ließ sich mit Hilfe der Clusteranalyse auf der Basis der Artenabundanzen eine Aufteilung der Untersuchungsstationen in zwei große Cluster durchführen. Zusätzlich waren zwei kleinere Cluster abzutrennen, die sich aus wenigen Stationen zusammensetzten (Abb. 12a). An den Stationen des großen westlich gelegenen Clusters 1 dominierten die Arten Nucula nitida und Spiophanes bombyx, an denen des Clusters 2 Abra alba und Scalibregma inflatum. Von Cluster 2 unterschied sich Cluster 3 durch eine hohe Dominanz von Scoloplos armiger und erhöhte Anteile von Eteone longa. Cluster 4 hatte erhöhte Individuenzahlen von Eumida punctifera, Pholoe minuta und Photis reinhardi.

Für April 1981 ergab sich ein anderes Bild über die Zusammengehörigkeit der Stationen (Abb. 12b). Hier gehörten die meisten Stationen in ein großes, zentrales Cluster 1, gegen das ein kleineres Cluster 2 abzugrenzen war. Ferner waren noch 5 weitere kleine Cluster an den Rändern des Untersuchungsgebietes nachzuweisen, die aber nur je eine Greiferprobe von zwei Stationen zusammenfassen. Die dominanten Arten in Cluster 1 waren Diastylis rathkei, Nucula nitida und Nephtys hombergi. Die Stationen des Clusters 2 unterschieden sich von denen des Clusters 1 durch das Auftreten von Abra alba und niedrigeren Abundanzen von Diastylis rathkei. Cluster 3 (aus je einer Parallelprobe von zwei Stationen) besaß eine ähnliche Artenzusammensetzung wie Cluster 1, nur fehlte hier Diastylis rathkei. Die übrigen Cluster waren auf Grund der Lage am Steilhang zur Elbemündung und nach Westen hin stark variierenden Einflüssen ausgesetzt, was die diverse Faunenzusammensetzung erklären könnte.

Aus den Ergebnissen der Clusteranalyse und der Rangkorrelation wurden die Artengemeinschaften zusammengestellt, die typisch für Cluster 1 bzw. Cluster 2 waren. Hierzu wurde für die betreffenden Cluster die Begleitfauna der drei dominanten Arten ermittelt. Die Begleitfauna 1. Ordnung waren die Arten, die mit den dominanten Arten positiv korreliert waren. Ebenfalls wurden für die Arten der Begleitfauna 1. Ordnung die Begleitfauna (2. Ordnung) ermittelt. Aus der Artenliste für die betreffenden Cluster wurden jene Arten als typisch erachtet, die eine negative Korrelation zu den dominanten Arten des anderen Clusters hatten. Die Arten der Begleitfauna 2. Ordnung, die eine negative Korrelation zu den dominanten Arten des eigenen Clusters hatten, wurden vernachlässigt.

Auffallend war die höhere Vielfalt der Taxa, die im Juli 1980 als typisch für Cluster 1 galten, während Cluster 2 nur von Polychaeta und Mollusca repräsentiert wurde. Als Kriterium für die Signifikanz einer Art für ein Cluster kann ihre Präsenz in dem Cluster gelten. Verglichen wurde die Präsenz der typischen Arten in den beiden unterschiedlichen Clustern (Tab. 4). Als typisch wurde eine Art bezeichnet, die in einem Cluster eine Präsenz von mehr als 50\%, in dem anderen Cluster aber eine Präsenz von weniger als $30 \%$ hatte. Aus den beiden Kriterien (1) negative Korrelation zu den dominanten Arten des anderen Clusters und (2) deutlich höhere Präsenz wurden die typischen Arten für Cluster 1 und 2 im Juli 1980 und April 1981 ermittelt (in Tab. 4 mit * gekennzeichnet).

\section{DISKUSSION}

In dem hier aufgesuchten Untersuchungsgebiet ließ sich ein negativer Einfluß des Klärschlamms auf die Artenzahl nachweisen, wie er schon an wenigen Dauerstationen 
im gleichen Seegebiet über den Zeitraum 1978 bis 1981 beobachtet wurde (Mühlenhardt-Siegel, 1988).

Im Juli 1980 war zwar die Artenzahl im Bereich der Klärschlammverklappung gegenüber den vorangegangenen Monaten erhöht, jedoch im Vergleich zu den weiter seewärts gelegenen Stationen sehr viel niedriger. Daher ist auf eine negative Wirkung des Klärschlamms auf die Artenzahl zu schließen. Der Artenverlust von Juli 1980 zum April 1981 war am Rand des Untersuchungsgebietes am größten. Das läßt sich so interpretieren, daß hier im Sommer Arten siedeln konnten, die im Winter absterben oder abwandern. Die Artenabnahme war im Umkreis des Klärschlammgebietes gering. Hier siedeln offensichtlich nur solche Arten, die auch im Winter das Gebiet nicht verlassen.

Die Änderung in der Biomasse von Juli 1980 bis April 1981 ist zum einen durch den Zusammenbruch der hohen Populationsdichte einzelner Arten, zum anderen durch eine Zunahme der häufigen Art Nucula nitida bedingt. Aus den Abundanzen der gefundenen Arten ließ sich mit Hilfe der Clusteranalyse das Untersuchungsgebiet in zwei große Cluster unterteilen, die sich als zwei unterschiedliche Assoziationen erklären ließen. Bemerkenswert war die Diversität in den beiden Clustern. Sie war im Juli gering in Cluster 2 und höher in Cluster 1. Im April waren die Verhältnisse umgekehrt, vermutlich verursacht durch eine hohe Dominanz von Nucula nitida und Diastylis rathkei in Cluster 1. Die Verschiebung der Isolinien mit geringster Evenness nach Nord-Westen in der Zeit vom Juli 1980 bis April 1981 läßt sich möglicherweise mit dem Strömungsbild der Deutschen Bucht in Einklang bringen. Nach Mittelstaedt \& Soetje (1982) soll ein Wirbel im Bodenwasser des Untersuchungsgebietes bei bestimmten Windrichtungen auftreten. Dieser könnte eine Drehung des "Hakens" in nördliche Richtung bewirken. Nach Ramming (1968) und Mittelstaedt (1980) sorgen die Restströme im gleichen Gebiet für eine Verdriftung nach Nord-Westen.

Wie es zu einer Ausbildung der zwei oben erwähnten Assoziationen kommen kann, soll im folgenden dargelegt werden. Nach Lewis (1978) kann die Artenzusammensetzung durch eine dominante Art bestimmt werden, wie für das Untersuchungsgebiet ebenfalls vermutet wurde. Die dominanten Arten während der vorliegenden Untersuchung waren Nucula nitida (Detritusfresser, langlebig) und Abra alba (Detritusfresser, hier kurzlebig). Der Zeitpunkt des Larvenfalls ist nach Thorson (1957) von großer Bedeutung für die Struktur einer Germeinschaft. Im Untersuchungsgebiet der Deutschen Bucht wurde der Larvenfall für den Frühling und Frühsommer angenommen, begründet durch das Antreffen der ersten Juvenilen in den Monaten April bis Juni (MühlenhardtSiegel, 1985). Caspers (1980) gibt den Frühsommer als Zeitpunkt für das Auftreten der Jungtiere der meisten Vertreter aus der Abra-Gemeinschaft der Deutschen Bucht an. Vermutlich auf Grund hydrographischer Bedingungen, wie der Wassererwärmung im Frühjahr (Boolootian, 1964; Thorson, 1966; Gerlach; 1972), liegt das Auftreten der juvenilen Tiere in den britischen Gewässern zeitlich vor dem in der Deutschen und Kieler Bucht. Es zeigte sich, daß die häufigsten Arten Diastylis rathkei, Nephtys hombergi und Nucula nitida zeitlich vor den Arten Abra alba und Pectinaria koreni siedeln. Wichtig ist, daß nach Rachor \& Salzwedel (1975) Nucula nitida eine benthische Entwicklung hat, die Larve also unabhängig von der Frühjahrsplanktonblüte ist und das Siedeln der Jungtiere in der Nähe der Elterntiere stattfindet. Abra alba besitzt eine pelagische Larve und kann von Elternpopulationen anderer Bereiche herantransportiert werden.

Nach Thorson (1957) haben die Larven vieler Arten die Fähigkeit, vor dem Siedeln 
Euklidische Distanz

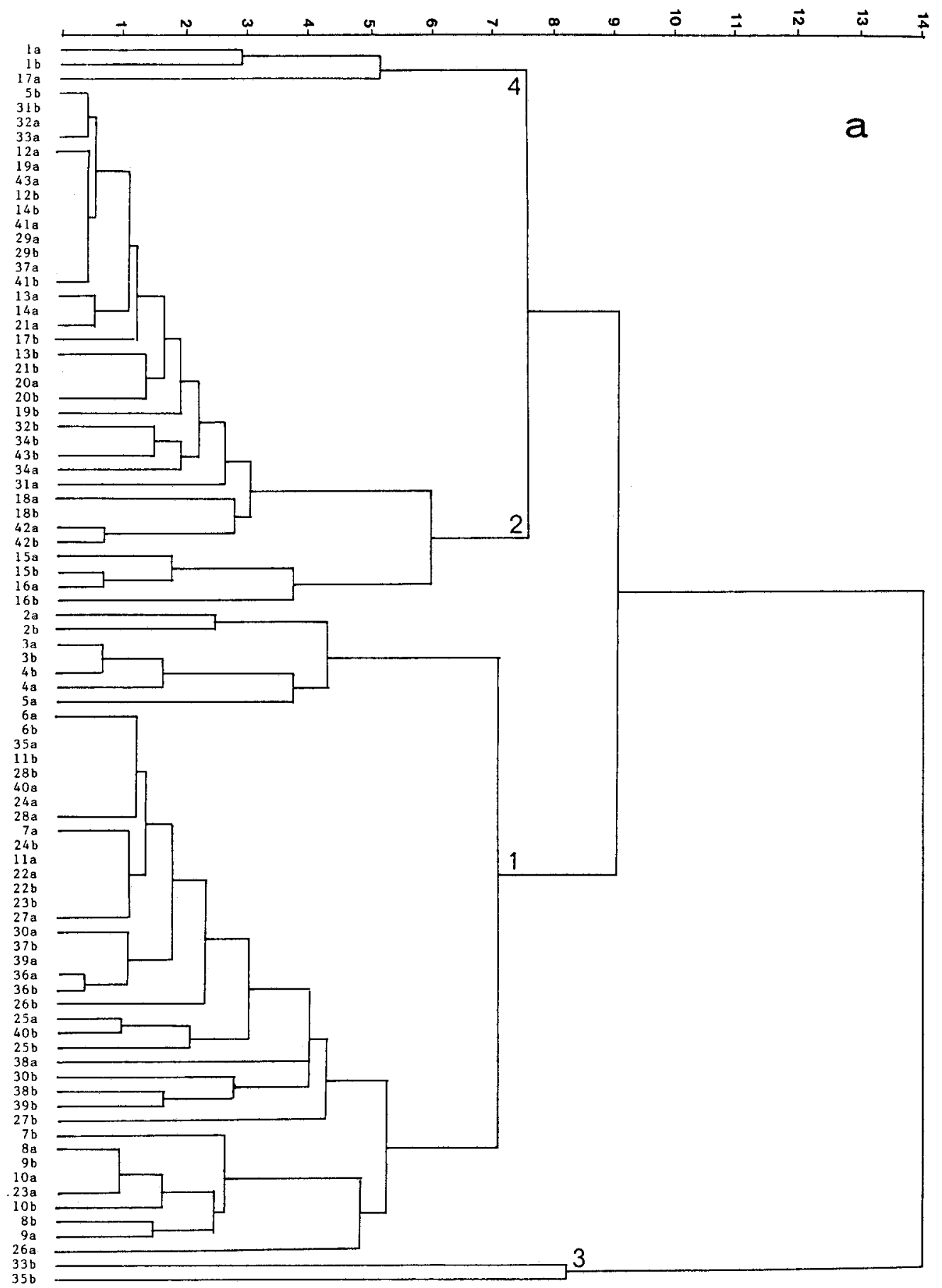




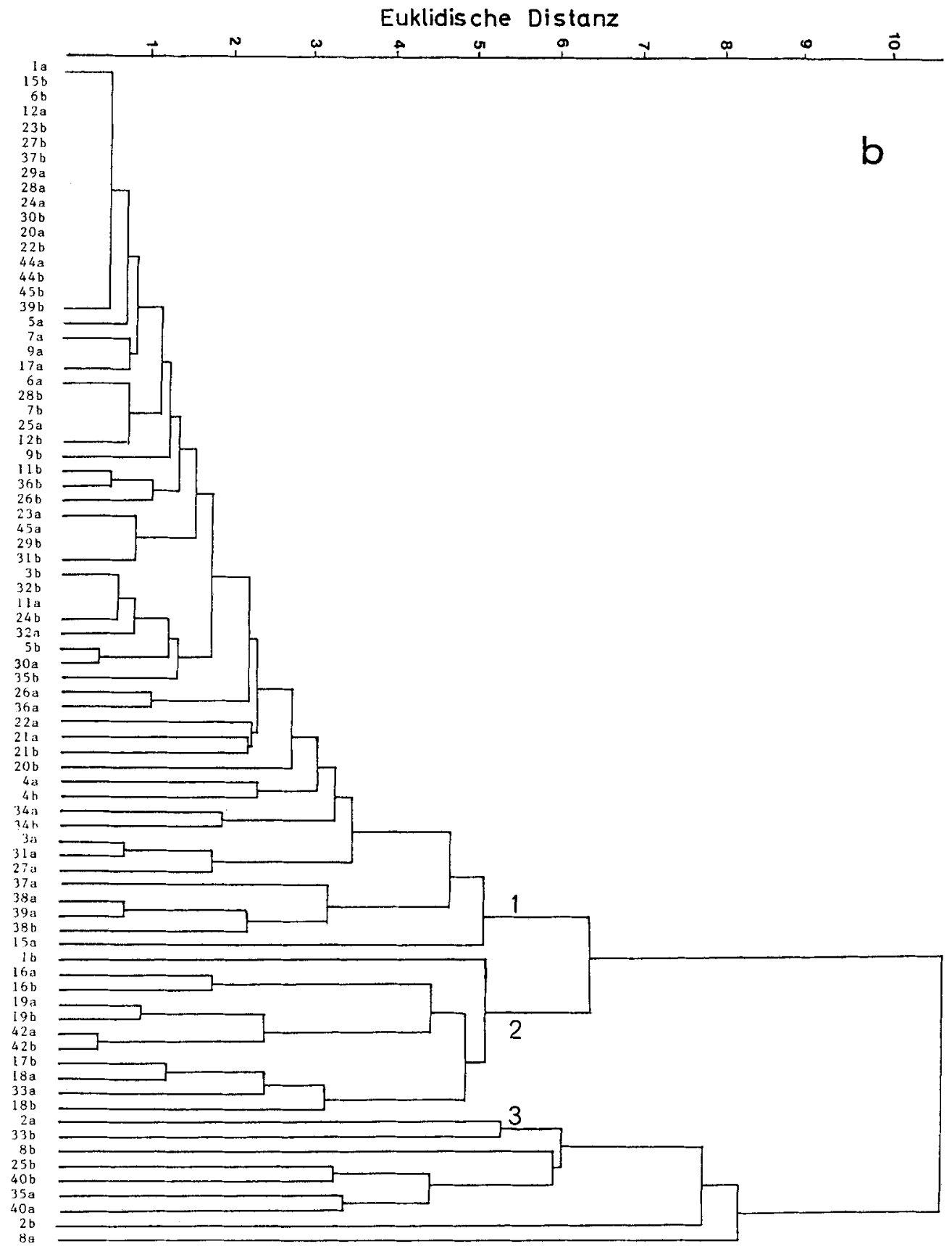

Abb. 12. Die Gruppierung der Stationen mittels der Clusteranalyse im Juli 1980 (a) und April 1981 (b) 
Tabelle 4. Vergleich der kennzeichnenden Arten für Cluster 1 und $2 .^{*}={ }_{n}$ typische Arten", $\left({ }^{*}\right)=$ Arten mit erhöhter Präsenz

\begin{tabular}{|c|c|c|}
\hline \multirow[t]{2}{*}{ Juli 1980} & \multicolumn{2}{|c|}{ Präsenz (\%) } \\
\hline & Cluster 1 & Cluster 2 \\
\hline Nucula nitida & 100 & 100 \\
\hline Diastylis rathkei & 100 & 100 \\
\hline Nephtys hombergi & 97 & 100 \\
\hline Scalibregma inflatum & 97 & 100 \\
\hline Eteone longa & 97 . & 90 \\
\hline Pholoe minuta & 97 & 87 \\
\hline Spiophanes bombyx & 94 & 57 \\
\hline Scoloplos armiger & 82 & 97 \\
\hline Ophiura albida & $79^{\circ}$ & 7 \\
\hline Abra alba & 76 & 97 \\
\hline Pectinaria koreni & 76 & 93 \\
\hline Ophelina acumenata & $76^{*}$ & 10 \\
\hline Ampelisca brevicornis & $73^{\circ}$ & 7 \\
\hline Halcampa cf. & $73^{*}$ & 20 \\
\hline Ampharete sp. & 68 & 17 \\
\hline Notomastus latericeus & $65^{*}$ & 23 \\
\hline Edwardsia sp. & $65^{\circ}$ & 23 \\
\hline Lineus sp. & $62^{*}$ & 17 \\
\hline Ophiura texturata & 59 & 96 \\
\hline Cerianthus lloydii & $59^{*}$ & 27 \\
\hline Diastylis bradyi & 56 & 17 \\
\hline Echinocardium cordatum & $56^{*}$ & 0 \\
\hline Goniada maculata & 47 & 3 \\
\hline Harmothoe lunulata & 47 & 27 \\
\hline Phoronis muelleri & $35\left(^{\circ}\right)$ & 0 \\
\hline Pariambus typicus & 35 & 17 \\
\hline Gattyana cirrosa & 32 & 33 \\
\hline Abra nitida & 32 & 0 \\
\hline Lanice conchilega & 32 & 20 \\
\hline Eudorella truncatula & 26 & 10 \\
\hline Aphrodite aculeata & 26 & 0 \\
\hline Tellina tenuis & 23 & $67^{*}$ \\
\hline Eumida punctifera & 23 & 27 \\
\hline Nephtys caeca & 20 & $63^{\circ}$ \\
\hline Corbula gibba & 18 & 0 \\
\hline Owenia fusiformis & 18 & 0 \\
\hline Argissa hamatipes & 15 & 3 \\
\hline Chone duneri & 12 & 0 \\
\hline Sphaeodorum flavum & 12 & 0 \\
\hline Macoma baltica & 9 & $63^{\circ}$ \\
\hline Nototropis swammerdami & 9 & 13 \\
\hline Thyasira flexuosa & 9 & 0 \\
\hline Montacuta ferruginosa & 9 & 0 \\
\hline Sagartia sp. & 6 & 7 \\
\hline Ensis directus & 3 & $67^{\circ}$ \\
\hline Magelona papillicornis & 3 & 33 \\
\hline Arenicola marina & 0 & 17 \\
\hline
\end{tabular}


Tabelle 4 (Fortsetzung)

\begin{tabular}{lcc|}
\hline April 1981 & & Präsenz (\%) \\
& Cluster 1 & Cluster 2 \\
\hline Nephtys hombergi & & \\
Diastylis rathkei & 98 & 82 \\
Nucula nitida & 96 & 54 \\
Ophiura texturata & 91 & 64 \\
Scoloplos armiger & 72 & 73 \\
Pholoe minuta & 60 & 91 \\
Abra alba & 46 & $73^{*}$ \\
Nephtys caeca & 43 & $64^{*}$ \\
Anaitides groenlandica & 38 & $64^{*}$ \\
Mysella bidentata & 33 & $64^{*}$ \\
Macoma baltica & 28 & 45 \\
Harmothoe lunulata & 24 & $73^{*}$ \\
Spiophanes bombyx & 21 & 45 \\
Ensis directus & 12 & $54^{*}$ \\
Abra nitida & 12 & $54^{*}$ \\
Echinocardium cordatum & $12\left(^{*}\right)$ & 0 \\
Eteone longa & $9\left(^{*}\right)$ & 0 \\
Montacuta ferruginosa & 7 & $73^{*}$ \\
Mya arenaria & $7\left(^{*}\right)$ & 0 \\
Bodotria scorpioides & 3 & 0 \\
Aphrodite aculeata & 2 & 0 \\
& 2 & 0 \\
\hline
\end{tabular}

das Substrat zu prüfen und nötigenfalls bei Ablehnung die pelagische Phase zu verlängern. Im hier behandelten Untersuchungsgebiet der Deutschen Bucht konnte gerade zur Zeit des Larvenfalls und des Auftretens der Jungtiere im Frühjahr keine Korrelation zwischen Sediment und Artenzahl oder Biomasse nachgewiesen werden (MühlenhardtSiegel, 1988). Hierdurch wurde auf eine Substratselektion erst später im Anschluß nach dem Larvenfall geschlossen. Die Beziehung zwischen Artengemeinschaften und Sedimenteigenschaften wurde schon öfter beobachtet (Nichols, 1970; Arntz et al., 1976; Coleman et al., 1978; Field et al., 1982; Tourtelotte \& Dauer, 1983).

Wie für das Untersuchungsgebiet in der Deutschen Bucht erstmals quantitativ nachgewiesen werden konnte, meiden die meisten Arten den Bereich mit hohen Siltund Tonanteilen im Sediment. Gleiches wurde auch für Seegebiete um Großbritannien (Holme, 1949; Buchanan et al., 1978; Warwick \& Uncles, 1980), Südafrika (Field, 1971; Christie, 1975) und Kanada (Hughes \& Thomas, 1971) beschrieben.

Remane (1964) hält einen hohen Anteil an organischer Substanz, wie sie meist in feinpartikulären Sedimenten vorkommt, auf Grund der Sauerstoffzehrung für nachteilig für die Bodenfauna. Nur wenige Gruppen bevorzugen die feinsten Sedimente. Hierzu gehört nach Cassie \& Michael (1968) Pectinaria, was die vorliegende Untersuchung bestätigt. Abra alba zeigte differierende Korrelationen zum Sediment im Verlauf des Untersuchungszeitraums, was mit dem oben genannten späten Larvenfall zu begründen ist. Danach waren die Gebiete mit hohem Anteil der gröberen Partikel von Nucula nitida und Nephtys hombergi besetzt, so daß Abra alba auf den weichen Schlick ausweichen mußte. Es wurde gezeigt, daß im Juli 1980 vor allem in diesem Gebiet der pH-Wert einen 
Einfluß auf die Besiedlung des Sediments hatte (Mühlenhardt-Siegel, 1985), was durch mögliches Vorhandensein säurebildender Mikroorganismen zu begründen ist. Den Einfluß der Bakterien auf die Siedlung von Makrofauna beschreiben ebenfalls Woodin (1976), Gerlach (1978) und Jumars \& Nowell (1984). Nach Hickel (1969) besteht zwischen Bakteriengehalt und organischer Substanz im Sediment bzw. feinen Partikeln (Mann, 1982) ein positiver Zusammenhang. Im Untersuchungsgebiet der Deutschen Bucht beeinflußten im Juli 1980 also zwei Faktoren die Substratselektion von Abra alba; Raumkonkurrenz und eine gute Nahrungsbasis. Beide Faktoren bewirken ein Ausweichen der Art auf ein Sediment, das nach den Ergebnissen aus dem Jahr 1979 nicht bevorzugt wird. Das Verbreitungszentrum von Abra alba lag im gleichen Seegebiet in früheren Jahren weiter westlich (Stripp, 1969a). Nach Figge (1981) und den eigenen Ergebnissen hat sich das Schlickgebiet im Vergleich zu den Werten von Stripp (1969b) nach Osten ausgedehnt. Ob die Raumkonkurrenz oder das Vorkommen von Bakterien in besonders feinen Sedimenten auf das Verteilungsmuster von Abra alba den größten Einfluß hatte, müßte geklärt werden.

Caspers (1979) beschrieb eine negative Korrelation zwischen Abra alba und Nucula nitida, während Glemarec (1973) beide Arten als typisch für weiche Sedimente angab. Die negative Beziehung zwischen Abra alba und Nucula nitida, die sich in der vorliegenden Untersuchung abzeichnete, läßt auf eine interspezifische Konkurrenz schließen, die nach Glemarec \& Menesguen (1979) vor allem in instabilen Lebensräumen groß ist.

Die Beziehungen der Arten untereinander war im Juli 1980 deutlicher als im April 1981 nachzuweisen. Für April 1981 konnten nur wenige Korrelationen von Abra alba mit anderen Arten nachgewiesen werden, was vermutlich auf das seltene Auftreten dieser Art zurückzuführen ist. Ophiura texturata hatte im April 1981, anders als im Juli 1980, eine positive Beziehung zu Nephtys hombergi, Nucula nitida und Diastylis rathkei, was durch eine weite Verbreitung des Ophiuriden durch den Larvenfall zu erklären ist.

Auch die Wirksamkeit von Räubern kann nicht bezweifelt werden. Diese sind vermutlich für den Zusammenbruch der Abra-Population verantwortlich. Hierbei sind die adulten Tiere als Beute von Bodenfischen anzusehen (Arntz, 1971; Arntz \& Hempel, 1972 ; Arntz \& Brunswig, 1975), während die Jungtiere von Ophiuriden gefressen werden (Tyler, 1977; Hily, pers. Mitt.). So werden alle Altersstufen dezimiert. Eine Neubesiedlung durch Abra alba ist danach nur möglich, wenn (1) Larven aus einem Gebiet außerhalb des Untersuchungsgebietes im metamorphosereifen Stadium eintreffen, (2) das Gebiet frei von Konkurrenten ist und (3) die Räuber von Larven und Jungtieren fehlen bzw. dünn gesiedelt haben.

Durch die Klärschlammverklappung war Voraussetzung 2 gewährleistet. Voraussetzung 1 hängt stark von den hydrographischen Bedingungen wie Temperatur (Einfluß auf die Laichzeit und Dauer der Larvalphase) und Strömung (eventuell modifiziert durch Winde) ab. Die Siedlungsdichte der Räuber ist nicht abzuschätzen; nach Ziegelmeier (1970) treten 2 Jahre nach extrem kalten Wintern Massenentwicklungen auf, die durch Fehlen von Predatoren begründet werden. Diese Erklärung kann auch für die Abra albaMassenentwicklung gelten, da der Winter 1978/79 sehr kalt war.

Nach Petersen (1914), Remane (1940), Stripp (1969b) und Salzwedel et al. (1985) muß die vorliegende Gemeinschaft der Abra alba-Coenose zugerechnet werden. Eine Zuordnung zu den klassischen Bodentiergemeinschaften konnte mit dem vorliegenden Material jedoch nicht vorgenommen werden. Nach Stripp (1969b) wäre das westliche Cluster 
1 dem Zentralgebiet der Abra-Gemeinschaft zuzuordnen. Hier hatte aber nach eigenen Untersuchungen Abra alba die geringsten Abundanzen, die hier siedelnden Arten zeigten sogar negative Korrelationen zu Abra alba. Die dominante Art hier war Nucula nitida, die charakterisierende Art war Echinocardium cordatum. Das zentrale Gebiet der Abra alba Verbreitung lag weiter östlich in einem Bereich, der nach Stripp (1969b) der Macoma-Gemeinschaft zuzuordnen wäre. Laut Eisma (1966) besteht zwischen Abra alba und Macoma baltica eine positive Korrelation, was die eigenen Untersuchungen bestätigen.

Nach den Ergebnissen der vorliegenden Analyse war keine Abra-Gemeinschaft im Sinne von Petersen (1914) nachzuweisen. Die hier gefundene Assoziation mit maximalen Abundanzen von Abra alba stellt auf Grund der Korrelation zu anderen Arten eine Untereinheit der Macoma-Coenose dar. Das Verbreitungsgebiet der klassischen AbraGemeinschaft wurde durch eine Übergangsgemeinschaft zur Echinocardium-Gemeinschaft mit Nucula nitida als dominanter Art charakterisiert. Die Klassifizierung einer Benthosgemeinschaft in diskrete Einheiten ist nach Ansicht mehrerer Autoren (Mills, 1969; Maurer et al., 1978; Grange, 1979) nicht berechtigt. Hierfür gibt Mills (1969) die binomiale Verteilung der Individuen und Arten entlang eines Gradienten als Begründung an, wobei diese unabhängig voneinander sein sollen. Es zeigte sich in der vorliegenden Untersuchung aber, daB gerade die Arten nicht unabhängig voneinander siedeln, wie die Wirksamkeit von benthischen Räubern und der Konkurrenzausschluß von Nucula nitida und Abra alba zeigte. Die Einteilung von Gemeinschaften nach typischen Arten erscheint unpräzise, wie durch das zeitweilige Fehlen der "Charakterart" Abra alba im Untersuchungsgebiet demonstriert wurde.

Die Untersuchung im großen Stationsnetz ließ eine räumliche Sukzession erkennen. Von einem artenarmen Zentrum im Bereich der Klärschlammverklappung aus war eine Zunahme der Artenzahl zu den Rändern des Untersuchungsgebietes hin zu verfolgen. In der Regel war die geringe Artenzahl mit einem Minimum an Individuenzahl und Biomasse korreliert. Durch die Massenentwicklung von Abra alba wurde diese Regel unterbrochen. Im Anschluß an dieses Gebiet mit hoher Biomasse folgte ein Areal mit toleranten Arten wie Nucula nitida, Diastylis rathkei, Nephtys hombergi, Corbula gibba und Abra nitida. Vereinzelt traten aber schon empfindliche Arten wie Echinocardium cordatum auf.

Nach Zerstörung einer Gemeinschaft unterscheidet man drei Sukzessionsstadien (Pearson \& Rosenberg, 1976, 1978; Pearson, 1981; Gray \& Pearson, 1982). Die Arten des ersten Sukzessionsstadiums sind gut an Störungen angepaßt und werden nach ihrer Überlebensstrategie als r-Strategen bezeichnet (Pearson, 1980; Rapport et al., 1981; Gray, 1982). Sie entsprechen den progressiven Arten 1. Ordnung, die Anger (1975) als durch Streß begünstigte Arten bezeichnet. In Bereichen mit hoher Dominanz von rStrategen ist eine hohe Variabilität der Individuenzahl und Biomasse auf Grund der Lebensgeschichte dieser Arten zu verzeichnen (Schwinghamer, 1983). Häufig treten rStrategen in instabilen Sedimenten mit hohem Wassergehalt auf (McCall, 1977) oder in Lebensräumen, die allgemein als inkonstant bezeichnet werden können (Boesch \& Rosenberg, 1981).

Nach Ursin (1982) sind labile und junge Systeme durch einen niedrigen Grad an Spezialisation und ein hohes Reproduktionspotential charakterisiert, das ein Überleben von genügend Larven möglich macht. Als Arten des ersten Sukzessionsstadiums gelten 
meist Polychaeta: Capitella capitata (Bagge, 1969; Rosenberg, 1972; Anger, 1977; McCall, 1977; Nichols, 1977; Eleftheriou et al., 1982), Scolelepis fuliginosa (Rosenberg, 1972), Tellina aegilis (McCall, 1977), Nereis diversicolor, Polydora ligni (Anger, 1977). Das zweite Sukzessionsstadium - im weiteren räumlichen oder zeitlichen Abstand von der Störung - ist durch Arten charakterisiert, die nur durch sehr starke Störungen beeinflußt werden, nach Anger (1977) indifferente Arten sind. Diese Arten überstehen leichte Verschmutzungen und gehören meist in die Gruppe der Bivalvia und Polychaeta (McCall, 1977): Scalibregma inflatum (Rosenberg, 1972; Pearson, 1981), Heteromastus filiformis (Parker, 1980; Rosenberg, 1972), Corbula gibba, Mya truncata, Cyprina islandica (Rosenberg, 1980), Nephtys hombergi, Scoloplos armiger, Diastylis rathkei (Parker, 1980), Glycera alba, Anaitides groenlandica, Pholoe minuta, Thyasira flexuosa (Rosenberg, 1972), Nucula nitida (Pearson, 1971). Die dritte Sukzessionsstufe ist durch sensitive Arten gekennzeichnet, die zu einer reifen Gemeinschaft tendieren und von Anger (1975) als regressive Arten bezeichnet werden. Besonders empfindliche Vertreter dieser Gruppe sind die Echinodermata (Rosenberg, 1972). Sie werden als erste bei Belastung eines Systems eliminiert (Pearson \& Rosenberg, 1976). Rachor \& Albrecht (1983) vermuten als Grund dafür eine Schädigung durch Sauerstoffmangel. Echinocardium cordatum gilt als sehr empfindlich (Rachor, 1982). Caspers (1979) berichtet vom Fehlen der Art Asterias rubens auf besonders weichen Sedimenten im Klärschlammverklappungsgebiet. Als sensitiv werden folgende Arten beschrieben; Echinocardium cordatum, Abra nitida, Amphiura filiformis, Ophiura albida und Ophiura texturata (Rosenberg, 1972), Eteone longa, Nephtys caeca, Scoloplos armiger (Anger, 1975), Bathyporeia sarsi (Anger, 1977).

Im Vergleich zu den genannten Sukzessionsstadien lag das Untersuchungsgebiet der Dauerstationen (Mühlenhardt-Siegel, 1988) bis Frühjahr 1980 im Bereich des „Ecotone Punkt" (Phase 2) nach Pearson \& Rosenberg (1978). Zur Zeit der Abra albaMassenentwicklung lagen die Schlickstationen im Übergangsbereich zwischen dem zweiten und dritten Sukzessionsstadium, wo nach Pearson \& Rosenberg (1978) das Biomassemaximum auftreten soll. Im großen Stationsnetz waren zwei Phasen des zweiten Sukzessionsstadiums zu erkennen. Die eine Phase zeigte ein frühes Stadium der zweiten Sukzessionsphase und war durch das Cluster 2 räumlich gekennzeichnet. Dieses Gebiet war durch das Vorkommen der Arten Scalibregma inflatum, Abra alba, Pholoe minuta und Nephtys hombergi charakterisiert. Das Gebiet, in dem Nephtys hombergi auftrat, leitete über zur zweiten Phase des zweiten Sukzessionsstadiums - hier gut beschrieben durch das Cluster 1 - mit den Arten Nucula nitida, Diastylis rathkei, Corbula gibba und Abra nitida. An einigen Stationen dieser Phase trat bereits Echinocardium cordatum auf, was einen Übergang zur dritten Sukzessionsphase andeutet. Das Untersuchungsgebiet in der Deutschen Bucht ist also in die zweite Sukzessionsphase einzuordnen, was nach McCall (1977) für küstennahe Lebensräume normal ist.

Danksagungen. Herrn Prof. Dr. C. D. Zander danke ich herzlich für die Diskussion des Manuskripts. Herrn Dr. H. Albrecht und seinen Mitarbeitern vom Deutschen Hydrographischen Institut, Hamburg, danke ich für die Hilfe bei der Probennahme, anregende Diskussion und die Möglichkeit, die Korngrößenanalyse durchzuführen. Diese Arbeitsgruppe überließ mir freundlicherweise die sedimentchemischen Daten. Herr R. Wais (Rechenzentrum der Universität Hamburg) und Herr Dr. O. Hüppop unterstützten die statistische Auswertung. Frau Dr. B. Hilbig half bei der Probennahme. Ihnen sei herzlich gedankt. 


\section{ZITIERTE LITERATUR}

Anger, K., 1975. On the influence of sewage pollution on inshore benthic communities in the south of Kiel Bay. Part 1: Qualitative studies on indicator species and communities. - Merentutkimuslait. Julk. 239, 116-122.

Anger, K., 1977. Benthic invertebrates as indicators of organic pollution in the western Baltic Sea. Int. Revue ges. Hydrobiol. Hydrogr. 62, 245-254.

Arntz, W. E., 1971. Die Nahrung der Kliesche (Limanda limanda) in der Kieler Bucht. - Ber. dt. wiss. Kommn Meeresforsch. 22, 129-183.

Arntz, W. E. \& Brunswig, D., 1975. An approach to estimating the production of macrobenthos and demersal fish in a western Baltic Abra alba community. - Merentutkimuslait. Julk. 239, 195-205.

Arntz, W. E. \& Hempel, G., 1972. Biomasse und Produktion des Makrobenthos in der Kieler Bucht und seine Verwertung durch Nutzfische. - Verh. dt. zool. Ges. 65, 32-37.

Arntz, W. E., Brunswig, D. \& Sarntheim, M., 1976. Zonierung von Mollusken und Schill im Rinnensystem der Kieler Bucht (westliche Ostsee). - Senckenbergiana marit. 8, 189-269.

Bagge, P., 1969. Effects of pollution on estuarine ecosystems. 1. Effects of effluents from woodprocessing industries on the hydrography, bottom, and fauna of Saltkällefjord (west Sweden). Merentutkimuslait. Julk. 228, 3-118.

Boesch, D. \& Rosenberg, R., 1981. Response to stress in marine benthos communities. In: Stress effects on natural ecosystems. Ed. by G. W. Barrett \& R. Rosenberg. Wiley, New York, 179-200.

Boolootian, R. A., 1964. Die Bedeutung abiotischer Faktoren für die Gonadenentwicklung und Fortpflanzung mariner Evertebraten. - Helgoländer wiss. Meeresunters. 10, 118-139.

Buchanan, J. B., Sheader, M. \& Kingston, F., 1978. Sources of variability in the benthic macrofauna off the south Northumberland coast, 1971-1976. - J. mar. biol. Ass. U.K. 58, 191-209.

Caspers, H., 1979. Die Entwicklung der Bodenfauna im Klärschlammverklappungsgebiet vor der Elbemündung. - Arb. dt. FischVerb. 27, 109-134.

Caspers, H., 1980. Long-term changes in benthic fauna resulting from sewage sludge dumping into the North Sea. - Prog. Wat. Technol. 12, 461-479.

Cassie, R. M. \& Michael, A. D., 1968. Fauna und sediments of an intertidal mud flat-a multivariate analysis. - J. exp. mar. Biol. Ecol. 2,1-23.

Christie, N. D., 1975. Relation between sediment texture, species 'richness and volume of sediment sampled by a grab. - Mar. Biol. 30, 89-96.

Coleman, N., Cuff, W., Drummond, M. \& Kudenov, J. D., 1978. A quantitative survey of the macrobenthos of Western Port, Victoria. - Aust. J. mar. Freshwat. Res. 29, 445-466.

Eisma, D., 1966. The distribution of benthic marine Mollusca off the main Dutch coast. - Neth. J. Sea Res. 3, 107-163.

Eleftheriou, A., Moore, D. C., Basford, D. J. \& Robertson, M. R., 1982. Underwater experiments on the effects of sewage sludge on a marine ecosystem. - Neth. Sea Res. $16,465-473$.

Field, J. G., 1971. A numerical analysis of changes in the soft-bottom fauna along a transect across False Bay, South Africa. - J. exp. mar. Biol. Ecol. 7, 215-253.

Field, J. G., Clarke, K. R. \& Warwick, R. M., 1982. A practical strategy for analysing multispecies distribution patterns. - Mar. Ecol. Progr. Ser. 8, 37-52.

Figge, K., 1981. Sedimentverteilung in der Deutschen Bucht. Deutsches Hydrographisches Institut, Hamburg, KtNr 2900.

Gerlach, S. A., 1972. Die Produktionsleistung des Benthos in der Helgoländer Bucht. - Verh. dt. zool. Ges. 65, 1-13.

Gerlach, S. A., 1978. Food-chain relationships in subtidal silty sand marine sediments and the role of meiofauna in stimulating bacterial productivity. - Oecologia 33, 55-69.

Glemarec, M., 1973. The benthic communities of the European North Atlantic continental shelf. Oceanogr. mar. Biol. 11, 263-289.

Glemarec, M, \& Menesguen. A., 1979. Functioning of a muddy sand ecosystem: seasonal fluctuations of different trophic levels and difficulties in estimating production of the leader macrofauna species. - In: Benthic production in the Bay of Biscay. Ed. by M. Glemarec. Inst. d'Etudes Mar., Brest, 1-11.

Grange, K. R., 1979. Soft bottom macrobenthic communities of Manukau Harbour, New Zealand. N. Z. Jl mar. Freshwat. Res. 13, 315-329. 
Gray, J. S., 1982. Effects of pollutants on marine ecosystems. - Neth. J. Sea Res. 16, 424-443.

Gray, J. S. \& Pearson, T. H., 1982. Objective selection of sensitive species indicative of pollutioninduced change in benthic communities. 1. Comparative methodology.-Mar. Ecol. Progr. Ser. 9, 111-119.

Hickel, W., 1969. Sedimentbeschaffenheit und Bakteriengehalt im Sediment eines zukünftigen Verklappungsgebietes von Industrieabwässern nordwestlich Helgolands. - Helgoländer wiss. Meeresunters. 19, 1-20.

Holme, N. A., 1949. The fauna of sand and mud banks near the mouth of Exe estuary. - J. mar. biol. Ass. U.K. 28, 189-237.

Hughes, R. N. \& Thomas, M. L. H., 1971. Classification of benthic samples from Bedeque Bay, an estuary in Prince Edward Island, Canada. - Mar. Biol. 10, 227-235.

Jumars, P. A. \& Nowell, A. R. M., 1984. Fluid and sediment dynamic effects on marine benthic community structure. - Am. Zool. 24, 45-55.

Lewis, J. R., 1978. The implications of community structure for benthic monitoring studies. - Mar. Pollut. Bull. 9, 64-67.

Mann, K. H., 1982. Sediment communities. In: Ecology of coastal waters. Ed. by K. H. Mann. Blackwell, Oxford, 183-210.

Maurer, D., Watling, L., Kinner, P., Leathem, W. \& Wethe, C., 1978. Benthic assemblages of Delaware Bay. - Mar. Biol. 45, 65-78.

McCall, P., 1977. Community patterns and adaptive strategies of the infaunal benthos of Long Island Sound. - J. mar. Res. 35, 221-266.

Mills, E. L., 1969. The community concept in marine zoology, with comments on continua and instability in some marine communities, a review. - J. Fish. Res. Bd Can. 26, 1415-1428.

Mirza, F. B. \& Gray, J. S., 1981. The fauna of benthic sediments from the organically enriched Oslo Fjord, Norway. - J. exp. mar. Biol. Ecol. 54, 181-207.

Mittelstaedt, E., 1980. Vorhersage der Drift von Meeresverschmutzungen in Küstengewässern. Seewart 41, 288-293.

Mittelstaedt, E. \& Soetje, K., 1982. Die Zirkulation in der Deutschen Bucht im August und September 1979. - Dt. hydrogr. Z. 35, 59-72.

Mühlenhardt-Siegel, U., 1981. Die Biomasse mariner Makrobenthos-Gesellschaften im Einflußbereich der Klärschlammverklappung vor der Elbemündúng. - Helgoländer Meeresunters. 34, 427-437.

Mühlenhardt-Siegel, U., 1985. Die Weichbodengemeinschaft vor der Elbemündung unter dem Einfluß der Klärschlammverklappung. Eine Analyse über Sedimentstruktur, Biomasse, Populationsdynamik. Diss., Univ. Hamburg, 198 pp.

Mühlenhardt-Siegel, U., 1988. Die Weichbodengemeinschaft vor der Elbemündung unter dem Einfluß der Klärschlammverklappung, Teil 1. - Helgoländer Meeresunters, 42, 37-65.

Nichols, F. H., 1970. Benthic polychaete assemblages and their relationship to the sediment in Port Madison, Washington. - Mar. Biol. 6, 48-57.

Nichols, J. A., 1977. Benthic community structure near the Woods Hole sewage sludge outfall. - Int. Revue ges. Hydrobiol. 62, 235-244.

Parker, J. G., 1980. Effects of pollution upon the benthos of Belfast Lough. - Mar. Pollut. Bull. 11, $80-83$.

Pearson, T. H., 1971. The benthic ecology of Loch Linnhe and Loch Eil, a sea-loch system on the west coast of Scotland. III. The effect on the benthic fauna of the introduction of pulp mill effluent. $-\mathbf{J}$. exp. mar. Biol. Ecol. 6, 211-233.

Pearson, T. H., 1980. Marine pollution effects of pulp and paper industry wastes. - Helgoländer Meeresunters. 33, 340-365.

Pearson, T. H., 1981. Stress and catastrophe in marine benthic ecosystems. In: Stress effects on natural ecosystems. Ed. by G. W. Barrett \& R. Rosenberg. Wiley, New York, 201-214.

Pearson, T. H. \& Rosenberg, R., 1976. A comparative study of the effects on the marine environment of wastes from cellulose industries in Scotland and Sweden. - Ambio 5; 77-79.

Pearson, T. H. \& Rosenberg, R., 1978. Macrobenthic succession in relation to organic enrichment and pollution of the marine environment. - Oceanogr. mar. Biol. 16, 229-311.

Petersen, C. G. J., 1914. Valuation of the sea. 2. The animal communities of the sea bottom and their importance for marine zoogeography. - Rep. Dan. biol. Stn 21, 1-44. 
Pielou, E. C., 1974. Population and community ecology. Gordon \& Breach, New York, 424 pp.

Rachor, E., 1982. Indikatorarten für Umweltbelastungen im Meer. - Decheniana Beih. 26, $128-137$.

Rachor, E. \& Albrecht, H., 1983. Sauerstoffmangel im Bodenwasser der Deutschen Bucht. - Veröff. Inst. Meeresforsch. Bremerhaven 19, 209-227.

Rachor, E. \& Salzwedel, H., 1975. Studies on population dynamics and productivity of some bivalves in the German Bight. In: Proceedings of the 10th European Symposium on Marine Biology. Ed. by G. Persoone \& E. Jaspers. Universa Press. Wetteren, 2, 575-588.

Ramming, H.-G., 1968. Ermittlung von Bewegungsvorgängen im Meere und in Flußmündungen zur Untersuchung des Transportes von Verunreinigungen. - Helgoländer wiss. Meeresunters. 17, 64-73.

Rapport, D. J., Regier, H. A. \& Thorpe, C., 1981. Diagnosis, prognosis and treatment of ecosystems under stress. In: Stress effects on natural ecosystems. Ed. by G. W. Barrett \& R. Rosenberg. Wiley, New York, 269-280.

Remane, A., 1940. Einführung in die zoologische Ökologie der Nord- und Ostsee. - Tierw. Nord- u. Ostsee, 1a, 1-238.

Remane, A., 1964. Die Bedeutung der Struktur für die Besiedlung der Meeresböden. - Helgoländer wiss. Meeresunters. 10, 343-358.

Rosenberg, R., 1972. Benthic faunal recovering in a Swedish fjord following the closure of a sulphite pulp mill. - Oikos 23, 92-108.

Rosenberg, R., 1980. Effects of oxygen deficiency on benthic macrofauna. In: Fjord Oceanograpy. Ed. by H. J. Freeland, D. M. Fanner \& C. D. Levings. Plenum Press., New York, 499 514.

Sachs, L., 1984. Angewandte Statistik. Springer, Berlin, 552 pp.

Salzwedel, H., Rachor, E. \& Gerdes, D., 1985. Benthic macrofauna communities in the German Bight. - Veröff. Inst. Meeresforsch. Bremerhaven 20, 199-267.

Schwinghamer, P., 1983. Generating ecological hypotheses from biomass spectra using causal analysis: a benthic example. - Mar. Ecol. Prog. Ser. 13, 151-166.

Stephenson, W., 1972. The validity of the community concept in marine biology. - Proc. R. Soc. Qd. $84,73-86$.

Stripp, K., 1969a. Jahreszeitliche Fluktuationen von Makrofauna und Meiofauna in der Helgoländer Bucht. - Veröff. Inst. Meeresforsch. Bremerhaven 12, 65-94.

Stripp, K., 1969b. Die Assoziationen des Benthos in der Helgoländer Bucht. - Veröff. Inst. Meeresforsch. Bremerhaven 12, 95-141.

Thorson, G., 1957. Bottom communities. In: Treatise on marine ecology and paleoecology. Ed. by J. W. Hedgpeth. Geol. Soc. Am., New York, 1, 461-534. (Mem. geol. Soc. Am. 67.)

Thorson, G., 1966. Some factors influencing the recruitment and establishment of marine benthic communities. - Neth. J. Sea Res. 3, 267-293.

Tourtellotte, G. H. \& Dauer, D. M., 1983. Macrobenthic communities of the Lower Chesapeake Bay. 2. Lymhaven Roads, Lymhaven Bay, Broad Bay and Linkhorn Bay. - Int. Revue ges. Hydrobiol. $68,59-72$.

Tyler, P., 1977. Sublittoral community structure of Oxwich Bay, South Wales, in relation to sedimentological, physical, oceanographic and biological parameters. - In: Biology of benthic organisms. Ed. by B. F. Keegan, P. O'Ceidigh \& P. J. S. Boaden. Pergamon Press, Oxford, 559-566.

Ursin, E., 1982. Stability and variability in the marine ecosystem. - Dana 2, 51-69.

Warwick, R. M. \& Uncles, R. J., 1980. Distribution of benthic macrofauna associations in the Bristol Channel in relation to tidal stress. - Mar. Ecol. Prog. Ser. 3, 97 103.

Woodin, S. A., 1976. Adult-larval interactions in dense infaunal assemblages: patterns of abundance.J. mar. Res. 34, 25-41.

Ziegelmeier, E., 1970. Über Massenvorkommen verschiedener makrobenthaler Wirbelloser während der Wiederbesiedlungsphase nach Schädigungen durch "katastrophale" Umwelteinflüsse. Helgoländer wiss. Meeresunters. 21, 9-20. 